\title{
GLAD!
}

Revue sur le langage, le genre, les sexualités

11 | 2021

Archives, genre, sexualités, discours

\section{Produire des archives lesbiennes : transmissions communautaires et connexions temporelles}

Producing Lesbian Archives: Community Transmissions and Temporal

Connections

Mathilde Petit

\section{OpenEdition}

Journals

Édition électronique

URL : https://journals.openedition.org/glad/3079

ISSN : 2551-0819

Éditeur

Association GSL

Référence électronique

Mathilde Petit, «Produire des archives lesbiennes : transmissions communautaires et connexions temporelles », GLAD! [En ligne], 11 | 2021, mis en ligne le 20 décembre 2021, consulté le 29 janvier 2022. URL : http://journals.openedition.org/glad/3079

Ce document a été généré automatiquement le 29 janvier 2022.

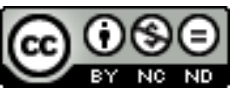

La revue GLAD! est mise à disposition selon les termes de la Licence Creative Commons Attribution Pas d'Utilisation Commerciale - Pas de Modification 4.0 International. 


\title{
Produire des archives lesbiennes : transmissions communautaires et connexions temporelles
}

\author{
Producing Lesbian Archives: Community Transmissions and Temporal \\ Connections
}

Mathilde Petit

Je tiens à remercier le comité de lecture de la revue, ainsi que les deux relecteurices anonymes de cet article. Je remercie tout particulièrement Pauline Gregory pour ses relectures et ses précieux conseils.

\section{Introduction}

1 Au milieu des années 1970, des mouvements militants lesbiens nord-américains comme la Lesbian Herstory Archive (LHA) de New York ont pris en charge la question de leurs archives et des mémoires communautaires. Parmi les réflexions imprégnant les pratiques collectives de mémoire et de production d'archives, l'enjeu des transmissions - qu'elles soient axées dans le temps ou dans l'espace - constituera le fil rouge de notre analyse. Cette dernière s'appuiera sur un corpus d'archives et de textes militants produits aux États-Unis entre 1975 et 1990. Les archives étudiées seront issues de revues et de newsletters rédigées sur cette période par la LHA (1), qui serviront de sources primaires pour l'analyse du discours tenu sur les productions d'archives et de mémoires lesbiennes. Elles seront mises en lien avec des textes militants et académiques qui permettront de contextualiser les archives et ainsi de les replacer dans un réseau plus large de réflexions lesbiennes-féministes. Si la majorité du corpus proposé est états-unien, nous pourrons faire appel à des autrices francophones (canadiennes et françaises), tout en rappelant que les contextes militants et nationaux demeurent distincts. Enfin, nous mobiliserons conjointement les analyses sociologiques $\mathrm{du}$ discours et des savoirs en les articulant avec les outils issus de l'historiographie. 
Nous mettrons ainsi en perspective les connaissances politiques et archivistiques des militantes sur leurs propres recherches, développées au cours de cette période extrêmement riche en termes de productions écrites et orales, encore relativement peu traduites en langue française. Nous choisissons l'angle de la transmission car il s'agit d'une part constitutive de la production d'archives lesbiennes, une démarche politique productrice de discours, de savoirs, d'échanges et de réflexions. Penser la transmission structure les pratiques, les manières d'agir et l'apprentissage de savoir-faire. Transmettre ou "faire passer» induisent par la signification même une action volontaire, consciente, qui participe à mettre en lien différentes temporalités. Dans le cadre d'une action collective, c'est aussi imaginer et construire des groupes passés, présents et futurs qui seraient liés temporellement, sans pour autant se figer ou devenir des unités fixes. Pour cet article, les termes existences et expériences lesbiennes seront privilégiés car usités par les militantes. En effet, les productions d'archives lesbiennes, qu'elles soient des réappropriations de matériaux anciens ou des productions d'archives en direct, s'appliquent à (re)créer et rendre visibles des traces d'existences, ainsi qu'à multiplier les traces d'expériences lesbiennes aussi bien pour investir les espaces et les temporalités historiques que pour rompre les isolements. Les deux expressions choisies incluent le terme «lesbienne » - qui a suscité de nombreux débats pour l'étude d'archives anciennes - dans la perspective de mises en lien d'expériences relationnelles analysées comme telles dans les perceptions culturelles et temporelles situées (Rupp 2013: 358) des discours politiques des militantes. Sans lui conférer une portée identificatoire élitiste et excluante (Zita 1981:173), nous nous référerons ici au fait que la « definition of lesbian we are looking for must include not only a descriptive and evaluative dimension but also a political dimension ${ }^{1}$ » (Zita : 173).

2 Notre argumentaire s'articulera autour de trois axes entremêlant trois temporalités : ce qu'a supposé cette transmission en termes de collecte ${ }^{2}$ d'archives anciennes et de pratique de réappropriations par les militantes; comment les productions d'archives en direct ont été conçues vers la transmission; et enfin, comment les connexions affectives et politiques créées entre les lesbiennes, leurs archives et leurs passés sont partie intégrante des processus de passation. Nous analyserons dans un premier temps comment ces pratiques archivistiques sont profondément liées aux discours politiques qui les traversent. Imbriqués aux luttes contre le patriarcat, les rassemblements d'archives participent aux pratiques de réappropriation communautaire, nécessaires pour la production de corpus à transmettre. Dans un deuxième temps, nous étudierons la manière dont les militantes ont suscité des productions d'archives en direct, afin de faire prendre conscience aux lesbiennes de la nécessité de communiquer leur propre expérience, afin de pallier les vides précédents et d'éviter les éternelles redécouvertes. Nous proposerons enfin d'étudier comment les productions de connexions temporelles ont facilité les processus d'identification nécessaires aux transmissions. Ces liens transhistoriques et multi-spatiaux à la portée performative s'inscrivent dans une perspective généalogique et générationnelle affectivement et politiquement concrète. Ils complètent ces actions en faveur d'une transmission des archives et passés lesbiens, qui se construisent en interaction avec les luttes communautaires contemporaines aux militantes. 


\section{Transmettre ses archives. Réappropriation des matériaux et production d'une conscience communautaire}

3 Pour comprendre comment les discours sur la transmission s'imbriquent aux productions d'archives lesbiennes, il faut saisir le contexte national et politique dans lequel s'inscrivent les militantes dont nous analyserons les écrits. Majoritairement issues de courants féministes, ces dernières ont parfois pris leurs distances avec des groupes ne favorisant pas l'émergence d'une réflexion sur les oppressions propres aux lesbiennes. Dans le contexte états-unien des années 1970 et 1980, une perspective critique lesbienne féministe ${ }^{3}$ se développe, de même que des militantismes lesbiens séparatistes ${ }^{4}$. Nous ne pourrons faire ici une présentation exhaustive (si tant est que cela soit possible) de l'ensemble des réflexions collectives et individuelles qui y ont éclos, mais en explicitant la manière dont les militantes se définissent politiquement au sein de ces débats aux multiples ramifications, nous pourrons entrevoir comment leurs positionnements influent sur la production d'archives et d'histoire lesbienne. Ce contexte de politisation de l'existence lesbienne sera donc abordé à partir des discours des militantes, pour éviter tout risque de raccourci inopportun ou simpliste. Sur ce même principe, nous emploierons le terme " archive » au sens qu'elles lui confèrent, alliant aussi bien le rassemblement de matériaux autour desquels se développent des pratiques documentaires que la production de nouveaux matériaux, de futures archives. Des pratiques qui émergent de deux constats: le vide archivistique des communautés lesbiennes et l'absence de transmission antérieure des matériaux ayant existé, au prisme des luttes contre le patriarcat et contre les perspectives hétérocentriques. D'une part, ces systèmes ont empêché la production d'histoires lesbiennes, en effaçant et tronquant les traces existantes. D'autre part, ils ont rendu difficile - voire impossible - la conception de l'idée de communautés au sein desquelles ces matériaux auraient pu être transmis.

\section{(Re)trouver ses archives, une quête empêchée : du constat des manques aux luttes contre les représentations patriarcales}

4 Les productions militantes d'archives telles que celles menées par la Lesbian Herstory Archive de New-York s'inscrivent contre un "historical void» ("vide historique ») (Bessette $2013: 28$ ) qui, à la fin des années 1970, n'est plus si vide qu'il n'y parait. Les luttes lesbiennes ont produit une multitude de matériaux : manifestes, enregistrements audios, revues. Ces derniers retracent des expériences militantes de lieux et d'époques données, qui témoignent de moments de vies, de luttes et de positionnements politiques : «We remembered a world of lesbian culture that had nourished us but that rapidely disappearing. We also knew, in this early heyday of lesbian publishing, that our presses and publishers were fragile undertakings, and we were concerned about preserving all their precious productions ${ }^{5}$ » (Nestle $1990: 88$ ). Ces productions écloses de manière foisonnante durant les années 1960-1970, menaceraient de disparaitre si aucune structure, aucun groupe ne s'attache à les préserver. Nestle, co-fondatrice de la LHA $^{6}$, emploie les termes de "préservation " et de "précieuses productions", deux conceptions prééminentes dans les newsletters. Le registre de la "fragilité », de la « disparition rapide», est mobilisé car les existences de ces groupes pouvaient être 
éphémères, dépendant des possibilités d'engagement de chacune des membres. Les matériaux pouvaient être perdus, entreposés dans des appartements soumis aux aléas des déménagements, des tris, des décès. Cet élan de conservation des traces récentes et la prise de conscience de leurs "pertes en direct ", s'accompagnent d'une réflexion sur les traces plus anciennes. Si tant de traces ont pu être créées en si peu de temps, pour quelles raisons les militantes qui ont investi ces missions archivistiques n'ont-elles pas trouvé les traces recherchées au moment où elles en avaient besoin? Ou tout au moins avec difficulté. Pour Nestle, la position spécifique des lesbiennes au sein d'un système patriarcal dominant en est l'explication :

When I first loved women in the late 50's, I was living the life of a colonized subject. I did not know it then. I thought it was accidental that I found no references in the surrounding culture to Lesbians creations. Sometimes believing the colonizers views of myself, I did not even search for markings because I knew we were not a people, just deviant sad wanderers, meeting in dark places ${ }^{7}$ (Nestle in LHA Newsletter 5 1979: $\left.3^{8}\right)(2)$

Cette analogie inscrit les lesbiennes dans une analyse plus large des systèmes d'oppression, comme "a people » qui n'a pas pu avoir accès à ses histoires, à ses cultures, ni même à la conscience de pouvoir se constituer en tant que communautés, au sein desquelles les savoirs se seraient transmis. Comme l'analyse Freeman : «Gays and lesbians have been figured as having no past [...] and crucially, no history as a distinct people 9 .» (2007: 162). Des représentations dominantes auxquelles Nestle "croit » jusqu'à ce qu'elle débute ses recherches. Similairement à Deevey, qui a contribué à la revue lesbienne séparatiste The Furies et y écrit «I looked at lesbians with all prejudice and fear I had learned as a heterosexual ${ }^{10} »(1972: 2)(3)$, Nestle adhère à une vision pessimiste de l'existence lesbienne, faite de « deviant sad wanderers ». Une expérience que l'on retrouve chez Martin et Lyon, deux membres fondatrices des Daughters of Bilitis ${ }^{11}$, lors de recherches infructueuses sur les lesbiennes dans l'histoire: "Instead of love, Martin read that her feelings for Lyon were a "perversion, a sign of psychopathology, a crime against nature and a sin against God "12.» (Bessette $2013: 29$ ) Deux problèmes s'entrecroisent: l'impossibilité à se penser et se construire comme peuple(s) avec histoire(s); et la recherche d'expériences similaires dont les seuls résultats renvoient aux représentations déviantes et pathologiques produites par un système patriarcal et hétérocentrique qui construit les lesbiennes en différentes ${ }^{13}$ (Wittig $2013: 62$ ).

\section{Critique lesbienne féministe et séparatisme lesbien : se penser « à part » et collectivement, une construction indissociable des processus de transmission communautaire}

6 Des représentations qui ont pu infuser au sein de mouvements féministes comme $\mathrm{NOW}^{14}$ (National Organisation for Women), dont Gottlieb et Kessler ${ }^{15}$ dressent un tableau très critique dans la revue off our back ${ }^{16}$ : "The oppression of women is considered a legitimate political and social concern whereas lesbianism is still seen as a psychological phenomenon and sexual perversion ${ }^{17}$.» (1980:9) Elles y contestent la minoration des présences lesbiennes et des discours les enjoignant à la discrétion, voire à ne pas employer un "word lesbian » encore pathologisé et délégitimant les causes féministes. Bien qu'elles considèrent le séparatisme lesbien comme une question controversée, elles constatent que son analyse radicale facilite la croissance de revues 
et presses lesbiennes féministes (1980:9). Un point de dissension y émerge : la naturalisation de l'hétérosexualité et des relations ${ }^{18}$ de domination qu'elle sous-tend en tant que système politique. Une question qui favorise la considération d'une existence lesbienne politique et historique, le terme «lesbienne » devenant dans une certaine acception synonyme de résistance aux relations de dominations hétéropatriarcales. Dénonçant l'obligation à l'hétérosexualité et l'absence de choix comme point aveugle des luttes féministes hétérosexuelles, Rich propose le «continuum lesbien » comme forme située et temporelle de résistance des femmes à ces relations (1981:42). Ce continuum comprend un ensemble de connexions réunissant des expériences de vie, d'oppressions et de résistances par des «regroupements des femmes dans toutes les structures raciales, ethniques et politiques» (1981: 42). Ces femmes n'ont pas nécessairement de relations affectives ou sexuelles : le lesbianisme est ici une mode de vie en résistance et toute femme refusant l'imposition des dominations patriarcales s'intègre au continuum lesbien. Cette analyse - souvent critiquée pour évacuer « the erotic $^{19}$ dimension from the concept lesbian ${ }^{20} »$ (Zita 1981 : 173-174) - comporte deux dimensions centrales : la continuité temporelle et spatiale d'une existence à part et leur construction comme "an act of resistance» (1981: 175). Si Nestle partage l'idée de connexion entre les expériences lesbiennes, sa définition du séparatisme l'encourage à penser les lesbiennes comme a people, ayant une existence historique distincte de l'histoire des femmes, y compris de celles en résistance aux systèmes patriarcaux: "These identities may be intertwined at times, but they are separate, distinct legacies, and at other times they may be in conflict ${ }^{21} . »(1987: 109)^{22}$. Un peuple qui commence à se penser comme tel et dont les recherches historiques et culturelles se discutent collectivement ${ }^{23}$. En 1975, la rubrique culture de la revue off our back diffuse une annonce invitant les lesbiennes à participer à un séjour isolé de trois jours aux alentours de Los Angeles, dans l'objectif d'échanger sur la «Lesbian History Exploration ». De multiples sujets et matériaux devaient être présentés : «story-telling, singing, dancing, historical fantasy, mythology, stories about lost women, research on known lesbians and lesbian communities of the past - anything that recreates lesbian history in written, aural, or visual form ${ }^{24}$. (4) Ces échanges collectifs autour de passés en «re-création» et le fait de se penser comme des identités (même fluctuantes) politiquement construites, tout cela apporte une dimension nécessaire aux processus de transmission et favorise le rassemblement d'archives ainsi que leurs circulations. Si les traces d'expériences sont à (re)découvrir, les connexions entre ces existences lesbiennes le sont également. Des connexions affectives et politiques, qui sont aussi importantes à produire que les histoires et matériaux à transmettre, dont certaines formes persistent mais auraient été effacées ou passées sous silence. Comment saisir ce qui a été, mais aussi ce qui aurait pu être ? Ce sont ces recherches individuelles semées d'embuches, de manques et de distorsions qui animent des " volontés de se souvenir ${ }^{25}$ " transformées en actions politiques pour se souvenir: «But the strongest reason for creating the archives was the silence of patriarchal history about us - women who loved women ${ }^{26}$.» (Nestle $\left.1990: 88\right)$

\section{Se réapproprier et construire ses passés : les analyses lesbiennes- féministes des archives et la " radical archiving »}

7 Déjouer silences et distorsions fait partie des savoirs qui se créent entre les militantes et universitaires et les matériaux qu'elles se réapproprient. Surtout quand leurs 
recherches portent sur des mises à jour de traces non perçues antérieurement. Ces analyses prennent particulièrement sens pour l'étude des œuvres d'autrices qui ont laissé des traces écrites et se concentrent sur deux points. D'abord le codage (ou coding) considéré comme une « lesbian ${ }^{27}$ survival technique " (Doughty 1979: 78). Et, dans le cas spécifique mais courant des échanges épistolaires des poétesses et écrivaines, les euphémisations ${ }^{28}$ réalisées a posteriori par leurs biographes ${ }^{29}$. Pour réinterpréter des matériaux partiellement exploités ou déformés, les militantes affirment la légitimité de critères d'analyse et d'expertise militante à articuler aux savoirs de l'analyse biographique. Une double perspective nécessaire pour déceler ${ }^{30}$ des indices plus sensibles $^{31}$ (« our standards, not theirs, should prevail $\left.{ }^{32} »\right)$ qui soit passent inaperçus, soit sont considérés comme une «insufficient evidence» («preuve insuffisante») (1979 : 77). En portant attention à ces traces, les historiennes et biographes lesbiennesféministes font émerger des fonds documentaires, faits d'ouvrages et d'échanges épistolaires, à même de constituer ce que les militantes appellent alors des archives lesbiennes. Des matériaux qui se heurtent au préalable aux mêmes dévaluations que ceux réhabilités par les historiennes féministes: «Women's achievements in any sphere tend to disappear from the historical record; women's achivements that take place in spheres valued only by women are almost sure to be invisible ${ }^{33}$.» (Doughty 1979 : 78). Les échanges épistolaires ou poèmes non édités du vivant des autrices sont associés à la sphère privée, ou encore au «non réel ». Les histoires romancées en disent parfois beaucoup sur leurs conditions d'existence mais entrent rarement dans le canon des matériaux légitimes faisant l'histoire, y compris académique. Et quand les autrices atteignent une reconnaissance publique, leurs biographies parviennent parfois tronquées. Faderman ${ }^{34}$, historienne spécialisée dans les études lesbiennes, critique notamment les processus de (ré)hétérosexualisation ${ }^{35}$ et l'effort effectué a posteriori pour effacer ou nuancer toute trace d'ambigüité dans leurs relations ou justifier leur célibat: "where biographers have been too scrupulous to bowdlerize, they have nevertheless managed to distort lesbian history by avoiding the obvious $^{36}$.» (Faderman 1979: 75). Face à des échanges trop explicites ou récurrents entre femmes, "éluder l'évidence » en distordant les matériaux s'est avéré un exercice précautionneux mais plus répandu que leur simple censure, par des pratiques de "chercher l'homme ${ }^{37}$ » (1979: 75) ou de "connections with the Great Men » (Doughty 1979 : 78). Si on ne peut pas affirmer que les biographes distordant ces récits aient eu conscience de priver des communautés de leur histoire, leur perspective hétérocentrique a contribué de fait à cet effacement, à la création et à l'entretien de représentations négatives qui leur étaient propres. Accéder à l'ensemble des corpus d'archives ${ }^{38}$ est une nécessité pour faciliter des relectures participant à la production d'une historiographie lesbienne placée dans la « peculiar historical position of having to prove the very existence of its subject matter ${ }^{39}$ " (Doughty 1979: 77). Militantes et universitaires doivent prouver que leurs recherches sont justifiées, tout comme la production de savoirs transversaux autour des existences lesbiennes. Pour Nestle, il s'agit alors de «say unhearable " ( dire l'inaudible»), jusqu'à ce que la présomption d'hétérosexualité soit elle aussi remise en cause : «[...] the burden of proof should be on the other interpretation - that they are not Lesbians ${ }^{40}$. » (Schwarz ${ }^{41}$ [Nestle] 1979:5) Souhaitant faire des années 1970 un temps où l'on établit l'expérience Lesbienne comme catégorie historique (Schwarz (Nestle) 1979: 5), elle réfute dans le même temps le fait de pouvoir "trust "historical understandings" or "academic institutions" 42 ", 
auxquels elle oppose sa vision radicale des archives ("radical archiving ») (Nestle : 1978) :

The Lesbian Herstory Archives must stay in its community, not out of parochialism but out of herstorical ${ }^{43}$ vision. We do not exist in historical understanding or academic institutions, though we travel incognito. We live on our homes, on the streets, in the bars, at our desks, at our jobs, with our children, in our groups, and we create our history every day. ${ }^{44}$ (Nestle $1978: 10$ )

8 L'omniprésence du «our » comme une stratégie discursive vient ici «établir une relation d'interlocution et de solidarité » (Michard 2009: 2). La séparation avec toute analyse historique rattachée au système patriarcal qui ne permet pas aux lesbiennes d'exister ("we do not exist ») apparait clairement dans ce discours, de même qu'une autre manière de faire histoire... ou de créer « our history » au travers de chaque action quotidienne. Vis-à-vis de ces réalités d'expériences, les analyses académiques sont mises à distance, à une période où justement les productions de savoirs légitimes se confrontent aux contre-savoirs militants ${ }^{45}$. Outre ces enjeux, Nestle met en avant la nécessité de conserver ces savoirs dans les communautés, compte tenu des problèmes d'accessibilité aux campus mais également de la sensibilité de contenus à protéger et préserver : "The archives should be housed within the community, not on an academic campus that is by definition closed to many women [...] If necessary the archives will go underground with its people to be cherished in hidden places until the community is safe ${ }^{46}$.» (Nestle 1978: 10). Pour elle, l'expérience singulière des lesbiennes (vies et militantismes) a conduit à un désir plus marqué de construire "a home of our Own " (5). En même temps que transmettre leurs archives aux lesbiennes qui souhaitent les consulter, le lieu où se trouvent ces archives doit être safe, accueillant, hospitalier. Elles ne doivent donc pas être confiées à des lieux susceptibles de n'en prendre pas soin voire de les détruire - tant que les possibilités d'existences des lesbiennes ne sont pas encore sûres. Cela ne signifie pas que les liens sont inexistants ou rompus : les archives lesbiennes sont également destinées aux chercheuses (universitaires ou non), afin de participer à la production d'un savoir féministe-lesbien, à partir de matériaux accessibles. Mais cela implique donc que les chercheuses viennent sur place, dans ce lieu d'archives communautaire, ce qui a été rendu possible dès 1976. Parallèlement à cela, la LHA enjoint l'envoi de tout travail universitaire réalisé par des étudiantes: "Students, we do not want your work to be lost. We want to keep on file all papers pertaining to all aspects of the Lesbian experience. Don't worry about style or grade. Send us a copy of your work so that all Lesbian may share your discover ${ }^{47}$.» (Nlt6 1980 : 3) (6) Cette fois-ci, le "all» prime dans le discours: les militantes souhaitent tout recueillir y compris tout travail de réflexion qui inclut des histoires lesbiennes et/ou est réalisé par des lesbiennes. Elles prennent en charge les passations, non seulement d'histoires passées vers le présent, mais aussi de savoirs en train de se construire, dont toutes les lesbiennes devraient bénéficier et qu'elles ne trouveraient pas ailleurs. Si certaines cherchent des références précises (raison pour laquelle le travail d'indexation est primordial ${ }^{48}$ ), d'autres vont être à la recherche de traces, dont le rassemblement préalable facilite la découverte. 


\section{Produire des archives en direct. De la lutte contre les silences à la transmission contemporaine : créer des matériaux, investir les espaces et les temporalités}

Pour les pratiques collectives d'archives, le rassemblement de traces d'existences s'accompagne de la production en direct de nouveaux matériaux, diffusables aux lesbiennes du présent et transmissibles à celles du futur. Sur différents supports, elles sont encouragées par les militantes à produire elles-mêmes ces traces, à se raconter. Mais la mise en récit de l'expérience lesbienne, tout comme le fait de se nommer comme telle, est moins évidente qu'il n'y parait et nécessite une réappropriation du langage, un travail pour reconstituer des non-dits et des manques. Le travail des militantes est également de leur faire prendre conscience de la nécessité de partager ce qui fait leur vie, ce qu'elles affirment être une responsabilité collective. Mettant en place des pratiques archivistiques moins " traditionnelles », elles s'emploient à tourner les archives vers l'avenir et vers des destinataires, remettant en question les temporalités linéaires.

\section{Multiplier les expériences lesbiennes et leurs traces matérielles}

10 Les analyses lesbiennes-féministes et les pratiques radicales des archives ont permis aux militantes de (re)constituer des passés identifiables et transmissibles aux lesbiennes en recherche de matériaux d'archives et de preuves d'existences. Dans une perspective de lutte très ancrée dans le présent ${ }^{49}$, elles ont conjointement encouragé la production en direct de (futurs) matériaux d'archives, représentatifs d'expériences présentes et transmissibles aux générations futures. En réponse aux silences et distorsions qui jonchent les existences lesbiennes, les militantes de la LHA ont enjoint chaque lesbienne à participer aux collectes :

We will collect and preserve ANY materials that are relevant to the lives and experiences of Lesbians: books, magazines, journals, new clipping (from establishment, feminist and Lesbian media), photos, bibliographies, herstorical information, tapes, films, diaries, oral herstories, poetry and prose, biographies, autobiographies, notices of events, posters, graphics, and other memorabilia and obscure references to our lives ${ }^{50}$. (Nlt4 $1978: 2$ ) (7)

11 Des matériaux qui participent à la production d'une archive sexuelle, dont certains éphémères comme les «notices of events " - constituent l'objet de prédilection des archives LGBTQI (Weeks ${ }^{11} 2019$ : 188). En dehors des canons habituels, ils documentent néanmoins «la vie intime, la sexualité, l'amour et le militantisme [...] dont il est difficile de faire la chronique en utilisant les seuls matériaux de l'archive traditionnelle» (2019: 189). Les militantes encouragent notamment leurs lectrices à produire des archives photographiques, un matériau qui manque aux communautés lesbiennes, mais dont le processus identificatoire et affectif est particulièrement fort :

The roots of the Archives lie in the silenced voices, the love letters destroyed, the pronouns changed, the pictures never taken, the euphemized distorsions that patriarchy would let past. To end of legacy of lost faces we are asking Lesbians all accross the country to send a photograph of themselves, friends, children, homes, pets, activities, to the Archives. Help us make sure our futur sisters will be able to see us $^{52}$. (Nlt5 $\left.1979: 4\right)$ 

matériaux non pas détruits ou effacés, mais «never taken» («jamais produits») : qui auraient pu exister si la production avait été possible (matériellement d'abord, mais surtout sans censure). Avoir accès à des images et des visages apporte une réalité quotidienne plus palpable à de (futures) archives, dont la portée affective les rend "aussi importantes que les mots» (Nestle 1990 : 91). Et d'autant plus qu'elles sont complexes à rassembler, engageant personnellement les lesbiennes plus que des productions écrites : «The books-newsletters-pamphlets are easy to come by but what we urgently need now are tapes-oral herstories-photographs of faces, homes, art works, friends-autobiographies, journals - letters - diaries ${ }^{53}$.» (Nlt3 1976:1) (8) Nestle revient en 1990 sur cette difficulté à convaincre les lesbiennes de se confier sur leurs existences :

Always we were asked, but you don't mean my work, my poems, my letters, my photograph? Always there was incredulity at our assertion that her life was the important one. But I had known this deprivation so searingly in my own life that it was a question that brought out all my fire and love - Yes, yes, you are the lesbian the archives exist for, to tell and share your story ${ }^{54}$. (Nestle $1990: 88$ )

Partager son histoire n'est pas chose aisée, surtout si elle ne semble pas présenter d'intérêt particulier pour les principales intéressées, dont les matériaux sont avant tout des objets de leur environnement, des traces de leur vie quotidienne ou des travaux qu'elles n'ont pas diffusés. Le rôle que se donne la LHA est de leur faire prendre conscience de l'intérêt que leur vie peut avoir, pour les autres lesbiennes mais également pour la passation temporelle de leur expérience. La pratique de la collecte de récits de vie, de témoignages enregistrés, a été particulièrement encouragée, dans de nombreux encarts ${ }^{55}$ et appels aux auto-enregistrements de soi-même et de ses connaissances. Ces pratiques reprenant les principes des collectes d'archives orales ${ }^{56}$, ont également permis de développer de nombreux savoirs collectifs. Apprendre à construire son récit, à mener un entretien et à l'enregistrer fait partie de ces apprentissages, dont l'objectif est la circulation des savoirs pour que chacune soit en mesure de constituer ses propres matériaux d'archives à partir d'expériences personnelles, qui tendraient à faire sens collectivement.

\section{Briser les silences pour exister : se réapproprier le langage pour construire des récits à transmettre}

Une pratique collective d'archive orale facilitée par les conseils des militantes, mais dont la réaction première d'incrédulité décrite ci-dessus par Nestle cache la culture d'un "silence tacite» constitutif d'histoires lesbiennes dépendantes d'éléments indescriptibles ou insaisissables (" elusive clue » : « indices insaisissables ») (Nlt6 1980 : 8). S'exprimer nécessite ici une réappropriation du langage, lutte primordiale des lesbiennes-féministes des années 1980. Elle a donné lieu à de nombreux échanges, dont ceux de Rich, Lorde ${ }^{57}$ et Stanley ${ }^{58}$ dans la revue Sinister Wisdom ${ }^{59}$ (1978) (9), dont la couverture porte les mots suivants : "Silence. Denial. Taboo. False-naming. Erasure. Encoding. Omission. Non-naming. Fragmentation. Lying.» (Rich 1978 : 24). Autant de termes à même de fragmenter existences, histoires et transmission :

I found myself telling another womon my coming out story, the story of how and when I had become a Lesbian, in all the senses in which I now use that word [...] I wanted her to understand me as I understand me, and I discovered in that telling 
that my life, my coming out, was a narrative of silences, the silence of denial, of self-hatred, of pain ${ }^{60}$ (Stanley 1978 : 5)

Ces silences ne sont donc pas toujours perceptibles au quotidien : c'est au moment de mettre en mots que celui-ci prend forme, imposant le constat suivant : si les lesbiennes ne sont pas en mesure de se définir et de transmettre leurs expériences au présent, les liens ne pourront pas se faire avec les générations futures. Pour Revillard, cette " répression silencieuse » est constitutive de l'oppression des lesbiennes et « constitue un obstacle pour la construction identitaire » individuelle et collective (2002: 176). Un « impensé social» (2002: 177) renforcé par l'absence de représentations (valorisantes) qui co-construit les parcours individuels, les comble de vides et de non-dits. Produire un récit de l'expérience du « devenir lesbienne » (le « become a Lesbian» employé par Stanley et notamment théorisé en France par Chetcuti) se heurte donc à cet obstacle indiscernable. Se nommer nécessite de sortir de "l'impossibilité d'être, puisque l'innommable est la conséquence de l'indiscernable, du méconnaissable. » (Chetcuti 2012 : 116). S'il est nécessaire de contextualiser la contemporaine évidence du récit du coming out (Chauvin, Lerch 2013 : 37-38), ce dernier est néanmoins un thème fédérateur qui peut servir de base d'échanges autour des expériences lesbiennes. C'est pourquoi les militantes de LHA ont réalisé une « lives tape collection », dans laquelle on découvre notamment la «Coming out story » de Mabel Hampton ${ }^{61}$, militante des droits des femmes et des lesbiennes afro-américaines $(10)^{62}$. Renseignant sur le contexte historique, politique et personnel des participantes, ces archives en devenir sont conçues pour apporter du soutien aux lesbiennes qui, après leur propre coming out, auraient besoin de "read and hear how the experience affected other Lesbians ${ }^{63}$ " (Nlt6 1980:2). La production de ce type de rubrique n'est pas anodine puisqu'elle oriente les matériaux d'archive orale vers des expériences fédératrices, qui de fait, apparaissent comme telles pour les lectrices et auditrices. Dans les discours, le caractère fédérateur ne doit pas se créer au détriment de la complexité des expériences lesbiennes, ce qui ne signifie pas néanmoins que l'inclusion recherchée ait fonctionné dans les pratiques ${ }^{64}$. La complexité des expériences - la "mosaic of the lesbian community" (Nestle 1990: 91) - est affirmée comme une richesse dont les lesbiennes doivent être fières, préférable à un récit universalisant et homogène : "a simplified, homogenized past will not be rich enough in ideas, inspirations, actions or images to nourish a diverse and embattled lesbian community of the future ${ }^{65}$ " (Nestle 1990 : 94). Une communauté future qui aura donc accès à de multiples matériaux que leurs prédécesseures auront contribué à produire dans un processus de "transformation of silence into language and action ${ }^{66}$ ", ce que Lorde considère comme "an act of selfrevelation" (1978: 15). Cette selfrevelation - indispensable à l'affirmation - permet de créer récits et traces de soi, et ainsi de briser «the millenia of silence about our lives " (Stanley 1978: 7). Par cette formulation, Stanley reconnait aux lesbiennes une existence dans le temps - une existence millénaire - reliée par ces silences. Il s'agit alors de créer «à partir de maintenant » des traces qui existeront pour le futur, selon les termes définis par leurs créatrices, et à transmettre comme tels : " As a Lesbian, I understand the importance of language in my life. With language, I can claim aspects of myself that I've denied, express ideas that have been suppressed and tabooed for a long time. With language, I can define my life as real, and I can act to change my life ${ }^{67}$.» (1978:7). 


\section{La prise de conscience archivistique : une participation collective aux productions d'archives destinées aux communautés présentes et futures}

16 Ayant établi pour elles-mêmes l'intérêt des archives dans les luttes lesbiennes, les militantes espèrent provoquer une prise de conscience archivistique («a process of archival consciousness raising ") (Bessette $2013: 24$ ) afin que chacune participe à cette production pour les futures chercheuses de passé : «[...] if the Archives is to end our generational loss of memory, every Lesbian woman must add her voice ${ }^{68}$. " (Nestle in Nlt5 1979: 3) Ajouter sa voix et participer aux productions d'archives enrichit ce que Kalcik (1975) nomme la « kernel story » ou « histoire du noyau », qui se construit via le partage d'expériences. Cette terminologie reprise par Bessette (2013:34) pour son étude de Lesbian/Woman (1972) - qu'elle considère comme une archive de partages d'expériences lesbiennes sur leurs recherches historiques infructueuses - est également pertinente dans notre contexte. En incitant à ce partage d'expériences, les militantes effectuent les connexions nécessaires à la prise de conscience (Bessette 2013 : 34). Prendre connaissance des newsletters a pu enjoindre d'autres lesbiennes à participer à ces productions d'archives et à les transmettre, afin qu'elles soient accessibles à d'autres. Afin d'éviter l'auto-censure et d'encourager au partage, la LHA privilégie une forme non panthéonisante de ces productions, faisant appel à toute forme de témoignage et de participation ( $"$ We try to avoid the hypocrisy of commemorating lesbians of the past while exiling their living representatives in the name of a selected herstory ${ }^{69}$. ( Nestle 1990: 91)). Prenons l'encart « a plea for coming out $^{70} »:$ : We need women to tell us if they should be a part of the Archives. Help us end the silences if for not now at least for the future ${ }^{71}$.» (Nlt4 1978:2). Le «help us », en adresse directe, fait appel à une responsabilité individuelle qui devient, par la prise de conscience militante, une responsabilité collective - voire communautaire - pour le futur. Les militantes impliquent ainsi de manière active chaque lectrice: "It is our responsability to validate the Lesbian experience for each other because it is through our collective rejoicing, reclaiming and renewing that our survival as a Lesbian community will be determined ${ }^{72}$.» (Nlt4 $\left.1978: 3\right)$.

17 Ces productions d'archives se singularisent des courants dits "traditionnels", les militantes concevant dès l'origine une transmission impliquant l'existence de destinataires. En effet, si on se réfère aux définitions de l'historienne Farge, les archives ne sont pas produites volontairement, n'ont précisément pas de destinataires (même potentiels ou fictifs) et n'existent «qu'au moment où on leur pose un certain type de question, et non au moment où on les recueille» (1997: 19). Cependant, il serait artificiel d'opposer une archive traditionnelle "professionnelle» à une archive militante sans prendre en compte les nuances existant dans le domaine archivistique. Concernant le rapport de l'archiviste à son objet, Klein (2019) sépare les courants dits «traditionnels " (caractérisés par un «ancrage dans le passé du geste producteur des documents ») de ceux se réclamant "de la pensée postmoderne de la fin du XXe siècle». Dans cette perspective, les archives ne sont plus des objets «accumulés naturellement et fixant le passé [...] mais elles sont la fabrique même d'un passé toujours en devenir » (Klein 2020). Fruits d'une construction sociale, les archives sont « un objet ouvert », dont l'exploitation leur confère un «lien privilégié avec l'avenir plutôt qu'avec le passé » (Klein 2019 : 92-93). Le rôle de relai temporel que souhaitent 
occuper les militantes se rapproche de celui revendiqué par les archivistes postmodernes, bien que ces derniers agissent en créant des fonds, tandis que les militantes produisent également des matériaux. Elles jouent donc un rôle actif et politique $^{73}$ dans la conception de leurs archives et des destinataires vers lesquelles la projection temporelle s'inscrit dans une double perspective : produire un héritage issu de matériaux et documents anciens qui font sens pour les lesbiennes, mais aussi produire en temps réel des traces d'existences pour des générations futures. Ce concept militant qui se « doit » d'être différent («Our concept of an archives must be different; we are different ${ }^{74}$.» (Nestle $\left.1978: 10\right)$ ) s'organise également autour de l'exploitation de matériaux existants, réappropriés afin de produire des savoirs collectifs : «Ces archives étaient dans une large mesure de nature matérielle, mais leur destination était également moins traditionnelle car elles étaient utilisées de façon active par les usagers pour des conférences, des débats, des présentations.» (Weeks 2019: 187-188). Il ne s'agit donc pas de transmettre un passé intouché, à mettre sous verre ou à réifier, mais bien de le rendre accessible afin qu'il prenne sens dans les réalités contemporaines. Ces échanges sur les matériaux sont en effet producteurs de savoirs collectifs et de significations sur la mémoire dans le présent (Weeks 2019: 187). Faire appel aux archives pour «reconfigurer le présent [est] une démarche primordiale dans toute stratégie de reconquête» (Danbolt 2010:6) Dans le cas des archives lesbiennes, cette reconfiguration s'expérimente conjointement dans la réappropriation de passé et dans la production d'archives contemporaines en vue de transmissions futures. Nécessairement politique, ce type d'archivage est doté d'un potentiel performatif qui agit sur les temporalités: "And already the archives has the record of how we are changing history ${ }^{75}$.» (Nestle 1978). Avoir accès à des archives d'expériences lesbiennes antérieures - (ré)analysées via des perspectives féministes-lesbiennes - a des effets sur les réceptrices présentes et inversement. Les connexions temporelles par le biais des archives ne sont pas unilatérales: elles produisent des allers-retours ${ }^{76}$ qui nécessitent de comprendre "la manière dont nous affectons le passé et sommes affectés par lui »(Danbolt 2010 : 10). Dans son étude sur les archives queer, Dinshaw (1999) travaille ce rapport à la " contemporanéité partagée », qui ouvre la possibilité de "créer des communautés partielles au-delà de la seule époque présente " (Danbolt 2010 : 10). Ces productions de liens temporels, ce travail du passé et du présent pour le présent et le futur (dans l'objectif aussi de combler des passés) se prête au jeu des historiographies queer. Comme l'affirme Orr (2017: 167): «The Lesbian Herstory Archives's forty-plus year journey has been a practice in queer potential [...] where radical archiving practices meet lesbian-centered politics for the purpose of realizing something more ${ }^{77}$. " Les archives lesbiennes doivent donc "réaliser quelque chose de plus ", produire une histoire passée et future plus foisonnante et enrichie des diversités lesbiennes, à laquelle chaque génération ajoute sa trace et contribue à changer l'histoire : « Once Lesbians have generations of herstory to experience, they will change history by the force of their presence ${ }^{78}$. (Nestle 1978). 


\section{Produire des connexions d'expériences lesbiennes: les enjeux des généalogies et des générations dans les processus de transmission}

18 La pensée de génération et la connexion à créer entre elles font partie intégrante de la production et de l'exploitation des archives. Ces attaches renforcent une perspective communautaire au sein de laquelle les lesbiennes seraient liées au travers de générations, s'incarnant dans des expériences de vie. Ces dernières se transmettent via la production de références liées au passé, par un héritage (re)créé de figures et de mythes historico-culturels qui véhiculent des expériences de luttes, des modes de vie, des existences dont la similarité fictive fait sens et agit politiquement. L'héritage culturel, par la production de généalogies créatives (notamment poétique) et le jeu des temporalités, facilite l'identification affective et la transmission. Cette dernière se construit également via la projection de liens familiaux concrets, entre des générations contemporaines les unes aux autres, à mettre en relation. Trois manières de transmettre s'entremêlent: une transmission militante au travers des enjeux de résistance aux normes patriarcales; une transmission culturelle par la connexion de pratiques artistiques; une transmission familiale par la projection de liens affectifs s'ancrant de manière plus concrète dans l'expérience quotidienne.

\section{Passés lesbiens et recherche de connexions généalogiques trans- temporelles : la transmission d'un passé de résistance dans un contexte de luttes contre le patriarcat}

La production d'archives d'expériences lesbiennes agit donc comme une ressource identificatoire forte. Elle s'affranchit des codes d'une archive traditionnelle en promouvant des allers-retours nécessaires entre les temporalités, fluidifiant en cela leur rapport à des passés en re(construction). Nous avons exploré jusqu'ici trois formes de production : réappropriation des passés; rassemblement de corpus; et production d'archives en direct. Pour qu'elles circulent et se transmettent, des connexions collectives doivent être construites ou renforcées. La projection générationnelle familiale parfois mobilisée («lesbian daughter») s'inscrit dans une pensée communautaire qui nécessite une conception et une politisation collective des liens d'intimité. Elle n'est certes pas propre aux années 1970 et 1980, mais prend un tournant collectif décisif dans le contexte de réflexion communautaire. La recherche généalogique peut aussi s'effectuer de manière individuelle, une quête qu'on retrouve par exemple dans des textes de la première moitié du XXe siècle. C'est dans ce cadre que Nealon analyse le rapport singulier entre existence historique et traçage généalogique des auteurices "gays men and lesbians ${ }^{79}$ " qu'il étudie (2001: 1). Cette connexion serait celle "d'enfant trouvé » (" foundling ») expérimentant un exil dont la pénibilité prend fin avec la re-création de solidarités nouvelles, au sein d'un "peuple » d'accueil. (2001: 1-2). Cet exil se rapproche de l'expérience d'anomie qui, après avoir induit une perte de repères, favorise la création de «formes nouvelles de relations humaines, d'autonomies qui ne sont pas celles d'une référence à des normes constituées, mais ouvertes sur une créativité possible » (Duvignaud 1986). La recherche de passé est donc ici profondément liée à la recherche de solidarité et de connexion créative. Au sein des pratiques archivistiques qui s'imbriquent aux luttes contre le 
patriarcat, cette production généalogique s'inscrit dans la transmission de cultures de résistance. Prenons deux figures antiques et/ou mythiques les plus mobilisées en Occident (et ce bien avant les années 1980) : les Amazones et la poétesse grecque Sappho. Les Amazones ${ }^{80}$, comme peuple de femmes guerrières, incarnent une forme de résistance contemporanéisée aux systèmes patriarcaux, et s'intègrent tout à fait au continuum lesbien de Rich. De multiples revues et groupes militants y font référence dont le collectif militant lesbien radical montréalais ${ }^{81}$ qui a fondé la revue Amazones d'hier, lesbiennes d'aujourd'hui ${ }^{82}$ (ALHA). Un nom qui établit clairement un lien temporel et généalogique et fait suite à la production d'un documentaire dont le visionnage est réservé aux lesbiennes. Par le biais d'entretiens, les militantes y interrogent leur entourage sur des points qui les interpellent: «comment on a commencé à être lesbienne? ?; " on est où? on vient d'où? on va où $?^{83}$ " (11) (Turcotte 2020). Les Amazones évoquent une lignée dont l'héritage représentatif et politique fait sens dans ces luttes lesbiennes des années 1980, d'autant qu'il fait aussi référence à un mode de vie séparatiste. Cavallaro - co-fondatrice de la LHA qui se définit également comme poète - écrit être "interested in exploring ways of creating an intellectual and agrarian Amazon lifestyle on the periphery of 'the system' with others Lesbians ${ }^{84}$." (Nlt1 $1975: 1)^{85}$ (12). Dans une période de développement du séparatisme lesbien y compris comme mode de vie alternatif, le qualificatif « amazon » renvoie à l'imaginaire collectif d'une vie de luttes menées entre femmes: «ces individus revendiquent de vivre en dehors de la "loi" de leur système de classe, comme l'a écrit Ti-Grace Atkinson dans Odyssée d'une Amazone » (Wittig 2013 : 94). D’autres références à la période antique sont mobilisées pour les titres revues lesbiennes ou de "serial media »: Cries from Cassandra ${ }^{86}$, The Furies, Amazon Quarterly, Echo of Sapho ou encore l'association Daughters of Bilitis ${ }^{87}$. Comme point d'ancrage généalogique, la figure de Sappho possède une place particulière. D'une part, elle est considérée comme une des premières lesbiennes dont la sexualité a été maintes fois réinterprétée, signe d'un lesbianisme comme «culture qui existe en dehors » du contrôle hétérosexuel et donc menaçante (Wittig 2013: 93). D'autre part, la destruction de ses poèmes crée le manque, un héritage empêché ${ }^{88}$ : " since the destruction of Sappho's poetry, our herstory has issued us a warning that we are among the disinherited ${ }^{89} »($ Nlt4 $1978: 3$ ). Les militantes affirment la position de Sappho comme première poétesse dont les écrits ont été détruits, annihilant toute possibilité d'héritage, (ré)affirmant sa place dans la généalogie avec l'évidence conférée à une donnée partagée. De fait, elles entretiennent et font perdurer des mythes historiques, culturels et politiques parfois déjà communs dans leurs contextes de référence ${ }^{90}$. L'affirmation de ces généalogies, nécessairement anachronique, possède une force politique performative qui inscrit celles qui l'expérimentent dans une temporalité millénaire, construite, choisie. Étant issue d'une construction évidente au même titre que d'autres formes qui tendent à être politiquement naturalisées - la production consciente de lien ne peut qu'être assumée et revendiquée dès lors qu'elle se pratique.

\section{Connexions créatives et production de généalogies littéraires et poétiques : lier les expériences lesbiennes}

Les figures historico-culturelles éloignées dans le temps permettent des réappropriations et rattachements plus fluides, qui inscrivent les lesbiennes s'y reconnaissant dans des passés historiques, mais également créatifs. Comme nous 
l'évoquions en première partie, les principaux matériaux anciens rattachés aux passés lesbiens sont majoritairement des écrits: épistolaires, littéraires ou fictifs. Ces productions sont constitutives de passés lesbiens et leurs matériaux composent tout un pan d'archives réappropriées à conserver et à mettre en lien. Pour les militantes de la LHA, ces littératures participent à la survie de chacune contre l'isolement ${ }^{91}$ : il s'agit donc d'en donner un accès rapide et facilité. La construction de liens affectifs et politiques est conçue comme indispensable, tout comme la production de cet héritage culturel historique qui participe à la cohésion et aux luttes contemporaines. Ces corpus créatifs permettent de créer des lignées d'écrivaines et notamment de poétesses. La culture poétique est ici une donnée mémorielle centrale, à produire aussi au présent. Loin de l'image élitiste qui peut lui être conférée, la poésie semble être un moyen d'expression fédérateur à investir politiquement. Au travers de ses nombreux poèmes, Lorde l'affirme comme moyen d'expression politique au sein duquel apparaissent les intersections de ses propres luttes : « as we reclaim our literature, poetry has been the major voice of poor, working class, and Colored women ${ }^{92}$ " (Lorde 1984: 116). Ayant inspiré de nombreuses lesbiennes, elle apparait avec Rich et Dickinson dans la « Poetry collection" (Nlt4 1978: 5), une indexation d'œuvres de 1923 à $1976^{93}$ (période déterminée par les matériaux archivés disponibles). Les autrices y sont classées par prénom, afin de contester la prééminence habituelle du patronyme. Toujours dans l'objectif d'éviter la panthéonisation, toute lesbienne est invitée à transmettre ses écrits afin d'apparaitre dans cette liste et de les rendre accessibles ${ }^{94}$ : «we ask Lesbian authors to send us copies of their work. Our funds are limited but our goal is to preserve Lesbians voices and to end the generational silence ${ }^{95}$.» (Nlt4 1978: 5) L'indexation de ces travaux - correspondant à un savoir professionnel qui se développe via la pratique militante - est un véritable outil pour faciliter l'accès aux archives passées et en devenir. Cette pratique est une production documentaire en ellemême: les catégorisations proposées incluent la vision archivistique des militantes, tant dans le classement des œuvres que dans les connexions qui se créent au gré des rassemblements, constituant des corpus aux significations nouvelles. Ces archives poétiques sont en effet assemblées car elles renseignent sur les existences lesbiennes et/ou sont produites par des lesbiennes, interrogeant la politisation de l'intimité des autrices et des archives intimes. Mais elles appartiennent parfois, pour les autrices ayant une résonnance publique, à des lignées déjà constituées. Créer des lignées créatives lesbiennes interroge les "généalogies habituelles [et] de l'histoire déjà écrite " (Alfonsi 2019: 6). Une démarche finalement proche des analyses lesbiennesféministes de Faderman et Doughty, visant à réintroduire les œuvres des autrices dans leur contexte de production. Alfonsi inclut notamment Cahun, dont l'œuvre redécouverte tardivement est affiliée au surréalisme et lui est presque entièrement attribuée, à la défaveur du couple artistique qu'elle formait avec sa compagne Marcel Moore ${ }^{96}$. (2019: 30) Remettre en perspective les artistes avec leur contexte de production politique, militant et personnel fait émerger de nouvelles accroches, plus transversales. La «vision téléologique de l'Histoire» est reconfigurée au profit de figures identificatoires non plus « en marche vers le progrès » mais « réservoir pour la création de lignées dynamiques qui établissent des contacts à travers le temps ». (2019: 47) Les archives littéraires et poétiques favorisent ces communications transtemporelles autant que les archives de partages d'expériences, s'affranchissant en partie d'une chronologie que Danbolt appelle le «temps straight». (2010: 8) (Re)prendre politiquement possession de la temporalité en revendiquant des 
connexions généalogiques choisies, fait ici partie des pratiques archivistiques lesbiennes. Cela ne signifie pas que les trois grandes temporalités passé(s)/présent(s)/ futur(s) ne sont pas mobilisées - elles le sont d'ailleurs beaucoup dans les discours mais elles sont fluidifiées par les interconnexions qui les relient entre elles.

\section{La construction générationnelle : filiations futures et préservation des mémoires à transmettre}

21 Ces contacts à travers le temps sont primordiaux dans les processus de transmission : ils rassemblent et rapprochent des expériences certes différentes, mais dont les liens affectifs se créent au travers d'allers et retours dynamiques entre passé et présent. Dans les discours des militantes de la LHA, ces liens affectifs prennent également forme dans une projection générationnelle très filiale (nous avons déjà noté dans d'autres extraits les termes («daughter» et «futur sisters») : «The Archives is both a library and a "family album": an attempt to preserve our living experiences beyond our generation and reconnect with our family of the past ${ }^{97}$.» (Nlt3 1976:1). La temporalité présente agit comme " a bridge to the past » (« un pont vers le passé ») (Orr $2017: 144$ ), qui conduit vers « notre » famille du passé, préservant conjointement passé et présent pour le futur. La préservation des traces présentes est d'autant plus prégnante s'agissant des « oral herstories from older lesbians» (« histoires orales des lesbiennes âgées ») (Nlt3 1976: 1). Une nouvelle fois, les militantes encouragent fortement les lectrices à devenir interviewers afin de " preserve the lives and voices of older women while they are still with us ${ }^{98}$ » (Nlt3 $\left.1976: 1\right)$. Et quand ces voix s'éteignent, la rubrique "In Memory of the voices we have lost» vient commémorer les "lost lovers and friends » et mettre fin aux épithètes « No Know Survivor » qui accompagnent les noms (Nlt7 1981 : 3) (13). Briser les silences sur les vies et ceux sur les morts s'entrecroise, pour des communautés qui - "as a people" - dénoncent la privation des rituels communautaires de deuils: "Often in newspapers, a euphemism for the death of a Lesbian is "there are no know survivors". This is not true. We are each other's survivors ${ }^{99} . »$ (Nlt7 1981: 3) En poursuivant au-delà de la vie les connexions générationnelles, les militantes participent à la production d'une interconnaissance intime, autour des expériences partagées de deuils silencieux et de situation d'isolement à rompre.

\section{Transmissions au présent : des archives créatrices de lien contre l'isolement et outils de luttes politiques contemporaines}

Ces pratiques archivistiques lesbiennes sont une nouvelle fois profondément politiques (" the Archives in the deepest sense is a political act ${ }^{100}$ » (Nlt5 1979:3)). Elles s'inscrivent dans les luttes présentes et survies quotidiennes, ayant pour ambition de briser les situations de détresse individuelle. Comme le souligne Bessette, le partage d'expériences au travers des pratiques de production d'archives incitent les lectrices à " contribute their own experience to the category, "reshaping the meaning of that individual experience" (Dubriwny 398) from a lonely ignorance to a shared indignation $^{101} »(2013: 34)$. Prendre conscience des expériences passées et présentes transforme un isolement vécu individuellement en vécu collectif socialement construit. 
Celui-ci se combat en rapprochant les lesbiennes temporellement et spatialement: "The lives of women who lived in different eras or cities or nations may touch each other. Individual lesbians and lesbian communities need never be fragmented or isolated again ${ }^{102}$." (Sturgis in Nlt5 1979: 4). Les militantes se font le relai des temporalités via ces pratiques archivistiques ancrées dans les luttes présentes. Une question de survie collective - qui comprend la survie de chacune - au quotidien : " our archives, our family album, our library, was not primarily for academic scholars but for any lesbian woman who needed an image or a word to survive the day ${ }^{103}$." (Nestle 1990 : 88) La mobilisation du champ lexical de la survie intensifie le recours aux archives pour connecter les lesbiennes à des images ou des mots salvateurs. Les archives s'inscrivent dans une perspective de soutien communautaire et dans la construction d'un «cross-generational, cross-cultural bridge » afin qu'aucun segment « of our community is to be singled out for societal repression ${ }^{104}$ » (Neslte 1990 : 91). Les discours mobilisent les archives dans un rapport très quotidien, proche des vies et conditions d'existences des lesbiennes, comme le détaille l'encart «How the archives can be of daily survival use » (Nlt6 $1980: 4)$.

Dans cette quête contre l'isolement, l'accessibilité aux matériaux d'archives est primordiale et il n'est pas rare que les archives soient déplacées d'une ville à l'autre. Les militantes de la LHA ont notamment produit un slide-show présenté dans de nombreuses villes des États-Unis. Composé d'images d'archives, il est conçu pour provoquer des échanges entre les lesbiennes et pour permettre aux militantes d'exposer leur vision de la pratique archivistique : « This slide-show became our major organizing tool, our most powerful way to work against feelings of cultural deprivation and personal isolation. It also allowed us to make our vision clear - what was a lesbian archives, how was it different from traditional archives how did it fit into the political struggles of our people? ${ }^{105}$ » (Nestle $\left.1990: 5-6\right)$ La rubrique « Tales from the road» fait état quant à elle d'un "diaries of a biker chick » : le voyage entre vingt-sept villes des États-Unis de Danzig - militante de la LHA - au cours duquel elle «bring the Archives to you " (" elle vous apporte les Archives»). (Nlt16 1996:4) Le journal rapporte de nombreuses anecdotes sur les rencontres faites dans villes traversées, donnant un aperçu des réactions et des présences lesbiennes sur le territoire états-unien. Le dynamisme du format de l'anecdote renforce la vision vivante des archives que les militantes souhaitent transmettre et le fait que les lectrices suivent les étapes contribue à enrichir le partage d'expériences. La connexion géographique est valorisée et les militantes montrent un intérêt particulier vers les lesbiennes vivant en dehors des grandes villes, avec lesquelles les contacts doivent être créés et maintenus. Ces déplacements militants étaient par ailleurs assez courants : on en retrouve chez les militantes montréalaises de ALHA, qui se sont beaucoup déplacées au Québec, aux États-Unis et en Europe - y compris à Paris dans les années 1990 - avec leur film éponyme évoqué plus tôt (Turcotte 2020). Par l'intermédiaire des archives, les militantes créent des liens géographiques physiques mais également de correspondance. Avec des rubriques comme "Rearch Questions ... Informations Needs ", elles assument un rôle de relai entre les recherches des unes et les réponses des autres (Nlt5 1979: 5). S'y rencontrent de multiples demandes de références : sur les "Spirituality and the Native American Lesbian through the Ages» et sur la vie de Christine de Suède ${ }^{106}$ par exemple. Mais on y trouve également des renseignements pratiques: adresses de groupes militants, services de santé alternatifs et conseils juridiques pour les gardes des enfants lors des divorces (Nlt6 1980 : 4). Leur implication 
dans ce rôle inscrit les militantes dans leur paysage militant contemporain. Par leur pratique archivistique, elles rompent avec l'impression passéiste que génère la première acception du mot «archive» pour participer activement aux luttes lesbiennes : «Our newsletter and displays in bookstores, libraries and gay community centres, our participation in demonstrations and marches, all make clear that a lesbian archives is a participant in the creation of culture and social change as well as a preserver of our people's story ${ }^{107}$. ( (Nestle 1990 : 92)

\section{Conclusion} production d'archives sont des voix qui annoncent l'autonomie et la « self possession » (Nlt5 1979: 3), éminemment politiques et intégrées aux luttes contemporaines. Confrontées au vide historique qui les précédait, les militantes ont activement participé aux collectes, préservations et productions de nouveaux matériaux dont elles ont cherché à faciliter les transmissions. Elles ont construit leurs pratiques archivistiques dans l'objectif de briser les générations de silences, briser les chaines des éternelles redécouvertes et des isolements inhérents à ces périodes d'exils durant lesquelles elles avaient en vain cherché des informations sur leur existence.

Se constituer en "peuple » ou en communautés politique et historique a participé aux transmissions d'archives, d'histoires redécouvertes et réappropriées, et des (contre ?)savoirs qui en découlent. Trois temporalités ont guidé leurs productions: « rediscovering our past, controlling our present, and speaking to our future » (Nlt1 1975 : 1). Une déclinaison retrouvée trois ans plus tard sous cette forme : " preserve our past »; " recognize the need of all Lesbians for a voice in the present and to ensure generational connection in the future » (Nlt4 $1978: 2$ ). Les deux versions s'entrecroisent et se complètent: le passé doit être redécouvert et préservé; le présent doit être contrôlé par les lesbiennes dont le besoin d'accès à ces matériaux doit être reconnu ; et enfin, les lesbiennes doivent parler et assurer une connexion intergénérationnelle afin de faciliter les processus de transmission. Les temporalités, loin d'être figées, sont mises en communication et influencent les unes et les autres leurs représentations. Les pratiques archivistiques qui s'y réfèrent sont productrices de nouvelles significations et interrogent sur la manière dont le passé peut servir au présent, et inversement. Des études queer des années 1990 ont privilégié d'autres interprétations, mettant en question ce «passé dont on peut se servir» au profit d'une analyse des «aspect du passé qui résistent à l'explication lorsqu'on s'en tient à l'identité » (Weeks ${ }^{108} 2019$ : 93). Interroger les significations des relations érotiques du passé et la manière « dont le désir féminin parvient à l'intelligibilité selon les époques " (2019: 94) ouvre la voie à d'autres subjectivités, plus nombreuses et plus inclusives. Ces dernières, vectrices d'expériences en dehors ou en négociation avec les normes sociales de leurs époques respectives, sont toujours susceptibles d'établir des connexions entre « des parcours de vie, des textes et d'autres phénomènes culturels qui, aujourd'hui comme autrefois, restent en dehors des catégories sexuelles. » (2019 : 95) L'établissement de liens transtemporels entre des expériences de vie reste un fil conducteur de ces pratiques et de ces analyses lesbiennes-féministes et queer, de même que la transmission des matériaux d'archives et des savoirs générés. La place majeure qu'occupent ces archives dans les militantismes lesbiens a également été questionnée, débattue, voire parodiée 
comme dans le film The Watermelon Women, réalisé en 1996 (Cvetkovich 2002). Sur un ton humoristique, Dune - la réalisatrice - pointe l'absence de production d'archives culturelles lesbiennes noires, dont la protagoniste principale est à la (vaine) recherche. Cette quête la conduit à un centre d'archive, dans lequel Cvetkovich voit une parodie de la LHA, comme « a form of respect and affection, demonstrating the important place of the archive in the lesbian popular imaginary ${ }^{109}$. " Des archives effectivement centrales, qui se sont imposées comme un instrument de libération (Weeks 2019 : 185) producteur de discours et de savoirs militants qu'il convient de diffuser, de partager et de transmettre, pour une passation générationnelle en profonde interaction avec les luttes présentes.

\section{BIBLIOGRAPHIE}

ALFONSI, Isabelle. 2019. Pour une esthétique de l'émancipation : construire les lignées d'un art queer. Paris : Editions B42.

BESSETTE, Jean. 2013. « An Archive of Anecdotes: Raising Lesbian Consciousness after the Daughters of Bilitis » Rhetoric Society Quarterly [En ligne], 43 (1) consulté le 08 mars 2021. URL : https://www.jstor.org/stable/41722462

BONNET, Marie-Jo. 1981. Un choix sans equivoque - Recherches historiques sur les relations amoureuses entre les femmes, XVIe-XXe. Paris : Denoel-Gonthier.

CAVALLARO, Sahli. 1975. «Biographical information from members ", LHA Newsletter $n^{\circ} 1$, consulté le $1^{\mathrm{er}}$ mars 2021. URL : https://lesbianherstoryarchives.org/content/uploads/2020/06/ News01.pdf

CALHOUN, Cheshire. 1994. "Separating Lesbian Theory from Feminist Theory ", Ethics [En ligne] 104 (3), consulté le 08 mars 2021. URL : https://www.jstor.org/stable/2381979

CHAMBERLAND, Line. 1989. « Le lesbianisme : continuum féminin ou marronnage ? Réflexions féministes pour une théorisation de l'expérience lesbienne » Convergences [En ligne], 2 (2) consulté le 06 mars 2021. URL : https://www.erudit.org/fr/revues/rf/1989-v2-n2rf1640/057563ar/

CHAUVIN, Sébastien \& LERCH, Arnaud. 2013. Sociologie de l'homosexualité. Paris : La Découverte.

CHETCUTI, Natacha \& GRECO, Luca. 2012. « La nomination de soi, entre catégorisation et intelligibilité : penser le genre » La face cachée du genre : Langage et pouvoir des normes. Paris : Presses Sorbonne Nouvelle. [En ligne], consulté le 10 juillet 2021. URL : https:// books.openedition.org/psn/3121

CORBMAN, Rachel. 2014. " A Genealogy of the Lesbian Herstory Archives, 1974-2014 » Journal of Contemporany Archival Studies [En ligne], 1, consulté le 10 mars 2021. URL : https:// elischolar.library.yale.edu/cgi/viewcontent.cgi?article $=1002 \&$ context=jcas CVETKOVICH, Ann. 2002. «In the Archives of Lesbian Feelings: Documentary and Popular Culture » Camera Obscura, 49 (17) : 1-147. 
CVETKOVICH, Ann. 2003. An Archive of Feelings : Trauma, Sexuality, and Lesbian Public Culture. Durham : Duke University Press.

DANBOLT, Mathias. 2010. « Anachronismes dérangeants : un sentiment historique avec N.O. Body » (tr. TRONC Hélène) [En ligne], consulté le 25 novembre 2020. URL : https://www.boudrylorenz.de/texts/

DEEVY, Sharon. 1972. «Such a nice girl » The Furies [En ligne] 1, consulté le 04 avril 2021. URL : https://repository.duke.edu/dc/wlmpc/wlmms01033

DEL MARTIN, \& LYON, Phyllis. 1972. Lesbian/Woman. San Francisco : Glide Publications.

DIDI-HUBERMAN, Georges. 2000. Devant le temps. Paris : Editions de Minuit.

DINSHAW, Carolyn. 1999. Getting Medieval : Sexualities and Communities, Pre- and Postmodern. Durham : Duke University Press.

DOAN, Laura. 2013. Disturbing practices : History, Sexuality and Women's experience of Modern War. Chicago : University of Chicago Press.

DOUGHTY, France. 1979. «Lesbian Biography, Biography of Lesbians » Frontiers : A Journal of Women Studies. Lesbian History [En ligne], 4 (3), consulté le 11 avril 2021. URL : https:// www.jstor.org/stable/3346155

DUBRIWNY, Tasha N. 2005. «Conscious-Raising as Collective Rhetoric : The articulation of Experience in the Redstockings' Abortion Speak-out of 1969 » Quaterly Journal of Speech 91 (4): 395-422.

DUVIGNAUD, Jean. 1986. Hérésie et subversion. Essais sur l'anomie. Paris : La Découverte.

FADERMAN, Lillian. 1979. « Who Hid Lesbian History? » Frontiers: A Journal of Women Studies. Lesbian History [En ligne], 4 (3), consulté le 08 mars 2021. URL : https://www.jstor.org/stable/ 3346154

FARGE, Arlette. 1997. Le goût de l'archive. Paris : Le Seuil.

FERGUSON, Ann. 1981. « Patriarchy, Sexual Identity and the Sexual Revolution », Signs [En ligne], 7 (1), consulté le 08 mars 2021. URL : https://www.jstor.org/stable/3173515

FREEMAN, Elizabeth. 2007. « Introduction. Special Issue: Queer Temporalities », GLQ: A Journal of Lesbian Gay Studies 13 (2).

GOTTLIEB, Rhonda \& KESSLER, Debra. 1980. « Where are all the militant dykes ? » Off Our Backs [En ligne], 10 (2), consulté le 1er mars 2021. URL : https://www.jstor.org/stable/25773195

KALCIK, Susan. 1975. « 'Like Ann's Gynecologist or the Time I Was Almost Raped': Personal Narratives in Women's Rap Groups » The Journal of American Folklore, 88 (347): 3-11.

KLEIN, Anne. 2019. Archive(s), mémoire, art. Eléments pour une archivistique critique. Québec (city) : Presses de l'Université de Laval.

KLEIN, Anne \& BESSON, Rémy. 2020. «Archive(s), mémoire, art : entretien avec Anne Klein », Entre Temps [En ligne], rubrique « Façonner » consulté le 31 aout 2021. URL : https://entretemps.net/archives-memoire-art-entretien-avec-anne-klein/

LORDE, Audre. 1978. " The Transformation of Silence into Langage and Action ", Sinister Wisdom (6) : 11-15.

LORDE, Audre. 1984. Sister Outsider : Essays and Speeches. Berkeley : Crossing Press. 
MICHARD, Claire. 2009. « Assaut du discours straight et universalisation du point de vue minoritaire dans les essais de Monique Wittig », Genre, sexualité \& société [En ligne], consulté le 05 décembre 2020. ULR : http://journals.openedition.org/gss/711

NEALON, Christopher. 2001. Foundlings : Lesbian and Gay Historical Emotion Before Stonewall, Durham : Duke University Press.

NESTLE, Joan. 1978. «About the Archives ", LHA Newsletter n5, consultée le $1^{\mathrm{er}}$ mars 2021. URL : https://lesbianherstoryarchives.org/content/uploads/2020/08/News05.pdf

NESTLE, Joan. 1978. « Radical Archiving: À Lesbian Feminist Perspective » Gay Insurgent (4) : 10.

NESTLE, Joan.1990. « The Will to Remember : The Lesbian Herstory Archives of New-York », Feminist Review. Perverse Politics: Lesbian Issues (34) : 86-94.

NESTLE, Joan.1993. « Excerpts from the Oral History of Mabel Hampton » Signs. Theorizing Lesbian Experience [En ligne], 18 (4), consulté le 29 avril 2021. URL : https://www.jstor.org/ stable/3174913

NESTLE, Joan. 2003 [1987]. « Voices from Lesbian Herstory » A Restricted Country. San Francisco : Cleis Press : 109.

ORR, Rebekah. 2017. The Ageing of the Archives : Community, Conflict, and Queer Potential at the Lesbian Herstory Archives. Thèse de doctorat en « Philosophy in Sociology ». Syracuse : Syracuse University.

PERRIN, Céline. 2004. «Sister Outsider d'Audre Lorde : la poésie et la colère », Nouvelles Questions Féministes, 23 (3) : 126-129.

REVILLARD, Anne. 2002. "L'identité lesbienne entre nature et construction », La Découverte, «Revue du MAUSS », [En ligne] 19 (1), consulté le 12 juillet 2021. URL : https://www.cairn.info/ revue-du-mauss-2002-1-page-168.htm

RICH, Adrienne. 1978. « The Transformation of Silence into Langage and Action », Sinister Wisdom (6) : 17-26.

RICH, Adrienne. 1981. «La contrainte à l'hétérosexualité et l'existence lesbienne ", Nouvelles Questions Féministes [En ligne] (1), consulté le 08 mars 2021. URL : https://www.jstor.org/stable/ 40619205

RUPP, Leila T. 2013. « Thinking About "Lesbian History" », Feminist Studies [En ligne], 39 (2), consulté le 08 mars 2021. URL : https://www.jstor.org/stable/23719048

SCHWARZ, Julie. 1979. "Questionnaire on Issues in Lesbian History » Frontiers : A journal of Women Studies [En ligne], 4 (3) « Lesbian History », consulté le 28 mars 2021. URL : http://www.jstor.org/ stable/3346142

STANLEY, Julia. 1978. " The Transformation of Silence into Language and Action », Sinister Wisdom (6) : 4-5.

STURGIS, Susanna. 1979. «In own write », LHA Newsletter n5. Consulté le $1^{\text {er }}$ mars 2021. URL : https://lesbianherstoryarchives.org/content/uploads/2020/08/News05.pdf

TURCOTTE, Louise \& COULOMBE Johanne. 2020. Entretien radiophonique réalisé dans l'émission «Toutes les lesbiennes en parlent », Consulté le 16 mars 2021. URL : https://www.youtube.com/ watch?v=a8Fh8fSTnR4.

WEEKS Jeffrey, 2019, Ecrire l'histoire des sexualités, Lyon : Presses universitaires de Lyon.

WITTIG, Monique. 2013 [2001]. La pensée straight. Paris : Editions Amsterdam. 
ZITA, Jacquelyn N. 1981. « Historical Amnesia and the Lesbian Continuum » Signs [En ligne] 7 (1), consulté le 08 mars 2021. URL : https://www.jstor.org/stable/3173516

\section{ANNEXES}

\section{CORPUS}

1. https://www.lesbianherstoryarchives.org/collections/newsletters/ (Consulté le 13 décembre 2020)

2. Newsletter LHA $n^{\circ} 5$ : News05.pdf (lesbianherstoryarchives.org) (Consultée le 15 décembre 2020)

3. The Furies (1): The Furies: Lesbian/Feminist Monthly (v. 1) / Women's Liberation Movement Print Culture / Duke Digital Repository (consulté le 30 avril 2021)

4. https://www.jstor.org/stable/25772079 (Consulté le 10 avril 2021)

5. https://lesbianherstoryarchives.org/content/uploads/2020/08/News16-1996.pdf (consulté le 10 avril 2021), sous-titre de la newsletter $n^{\circ} 16$ (1996)

6. Newsletter LHA $n^{\circ} 6$ : https://lesbianherstoryarchives.org/content/uploads/ 2020/08/News06.pdf (Consultée le 18 décembre 2020)

7. Newsletter LHA $n^{\circ} 4$ : https://lesbianherstoryarchives.org/content/uploads/ 2020/06/News04.pdf (consultée le 15 décembre 2020)

8. Newsletter LHA $n^{\circ 3}$ : https://lesbianherstoryarchives.org/content/uploads/ 2020/08/News03-1976.pdf (consultée le 13 décembre 2020)

9. Sinister Wisdom (6) : Sinister Wisdom 6_0.pdf (Consulté le 10 mars 2021)

10. http://herstories.prattinfoschool.nyc/omeka/document/SPW1132 (Consulté le 10 avril 2021).

11. https://www.youtube.com/watch?v=a8Fh8fSTnR4. (Consulté le 16 mars 2021)

12. Newsletter LHA $n^{\circ} 1$ : News01.pdf (lesbianherstoryarchives.org) (Consultée le 04 décembre 2020)

13. Newsletter $n^{\circ} 7$ : https://lesbianherstoryarchives.org/content/uploads/2020/08/ News07-1981.pdf (Consultée le 10 janvier 2021)

\section{NOTES}

1. Afin de faciliter la compréhension et la fluidité de la lecture, je proposerai en note de bas de page une traduction des extraits longs, en tentant au mieux de préserver les intentions des autrices. Ici la traduction proposée : "la définition de "lesbienne" que nous recherchons doit inclure non seulement une dimension descriptive et appréciative, mais également une dimension politique».

2. Le terme « collecte » se rapporte à la terminologie archivistique. Nous emploierons cependant plus régulièrement l'expression "rassemblement» au cours de ce développement, faisant référence au «to gather » (rassembler) ou «the gathering " (le rassemblement) employé par les militantes (voir par exemple Nestle 1990 : 87).

3. Élaborer une définition stricte du feminist lesbianism (perspective critique mobilisée par un grand nombre de militantes, activistes et universitaires et donc autant de développements analytiques), n'est pas chose aisée. Nous allons tout de même apporter certains éléments de compréhension et de contextualisation. La perspective critique lesbienne féministe affirme l'hétérosexualité comme institution politique et dénonce sa naturalisation, notamment au sein des militantismes féministes de cette période, ainsi que les multiples oppressions (collectives et 
individuelles) qu'elle fait peser sur les femmes (en tant que catégorie sociale construite). Le lesbianisme féministe encourage donc à dénaturaliser le système politique hétérosexuel et à comprendre comment il est devenu ce système oppressif, dans une perspective à la fois politique et historiographique. Adrienne Rich, Audre Lorde ou Lillian Faderman (dont nous analyserons des extraits dans cet article), mobilisent cette perspective qui nourrit nombre de travaux, notamment sur les possibilités d'une existence lesbienne dans l'histoire.

4. Le séparatisme lesbien s'intensifie également dans les années 1970 aux États-Unis. Conçu comme une stratégie parfois temporaire de retrait, il est aussi mobilisé comme mode de vie plus global (vie en communautés restreintes dans certains cas). Il s'appuie notamment sur un féminisme lesbien radical qui refuse la participation au régime politique hétérosexuel. À cette période, le séparatisme lesbien permet aux militantes de réfléchir sur leurs propres oppressions au sein du système politique hétérosexuel, mais également vis-à-vis de l'effacement des problématiques lesbiennes au sein de mouvements féministes (l'article «Separating Lesbian Theory from Feminist Theory" (Calhoun 1994) apporte par exemple un éclairage sur ces questions), et des mouvements homosexuels masculins. Il se construit aussi par le développement d'une culture lesbienne indépendante (bars, évènements culturels et librairies).

5. «Nous nous souvenions d'un univers de culture lesbienne qui nous avait nourries, mais qui disparaissait rapidement. Nous savions aussi, en ce début d'âge d'or de l'édition lesbienne, que nos presses et éditeurs étaient des entreprises fragiles et nous étions soucieuses de préserver toutes leurs précieuses productions. » [trad. de l'autrice]

6. Dans son article « The Will to remember : The Lesbian Herstory Archives of New-York » (1990), Nestle se définit comme marxiste et lesbian separatist. Elle forme avec Cavallaro et Stanley (sur lesquelles nous reviendrons au cours de cet article) un groupe lesbien séparé («Women's Caucus ») au sein de la Gay Academic Union, afin d'aborder les problématiques spécifiquement lesbiennes. Dès les premières réunions, la question des archives et de la préservation de l'histoire lesbienne est abordée et sera rapidement développée dans les mois qui suivront (Corbman 2014 : 7).

7. "Quand j'ai aimé les femmes pour la première fois à la fin des années 50, je vivais la vie d'un sujet colonisé. Je l'ignorais alors. J'ai pensé que c'était un hasard si je n'avais trouvé aucune référence aux créations lesbiennes dans mon environnement culturel. Croyant parfois aux conceptions des colonisateurs à mon propos, je n'ai même pas cherché de traces car j'étais persuadée que nous n'étions pas un peuple, juste de tristes et déviantes vagabondes, se rencontrant dans des endroits sombres. » [trad. de l'autrice]

8. Afin d'alléger les références, nous raccourcirons ensuite comme suit: Nlt5 1979. Nous indiquerons le nom de l'autrice quand celui-ci est connu: s'agissant de productions collectives, cela n'est pas toujours le cas. Le numéro de page (indiqué comme pour les autres références après la date de parution) correspond quant à lui au numéro de page du document numérisé.

9. «Les gais et les lesbiennes ont été considérées comme n'ayant pas de passé [...] et surtout, pas d'histoire en tant que peuple distinct. » [trad. de l'autrice]

10. "Je regardais les lesbiennes à travers tous les préjugés et la peur que j'avais appris en tant qu'hétérosexuelle.» [trad. de l'autrice]

11. Considérée comme la première association historique lesbienne aux États-Unis (fondée en 1955).

12. "Au lieu de l'amour, Martin a lu [au cours de ses recherches sur l'homosexualité féminine] que ses sentiments pour Lyon étaient une "perversion, un signe de psychopathologie, un crime contre nature et un péché contre Dieu". " [trad. de l'autrice]

13. Dans La pensée straight, Wittig analyse la construction d'un "autre-différent» comme «nécessité ontologique pour tout le conglomérat de sciences et de disciplines » qui constitue la pensée straight, dont l'histoire (Wittig 2013: 62). Tout groupe construit et assigné comme différent par le régime politique hétérosexuel, n'a pas le contrôle sur ses histoires(s), ses culture(s) et ses propres catégories. 
14. Organisme féministe fondé en 1966 aux États-Unis. Si Gottlieb et Kessler en dressent un portrait critique, certaines militantes lesbiennes y ont participé (Del Martin et Lyon par exemple), notamment dans un groupe de travail créé en 1973. Le fait que sa co-fondatrice Friedan ait été hostile à l'ouverture aux questions lesbiennes (Lavender Menace) a donné naissance au manifeste "The Woman Identified Woman » par les Radicalesbians en 1970, considéré comme un document fondateur du féminisme lesbien.

15. Se définissant toutes deux comme des lesbiennes féministes actives dans le «Women's Liberation Movement " pendant de nombreuses années.

16. Journal féministe radical états-unien paru entre 1970 et 2008.

17. "L'oppression des femmes est considérée comme une préoccupation politique et sociale légitime alors que le lesbianisme est toujours considéré comme un phénomène psychologique et une perversion sexuelle." [trad. de l'autrice]

18. Qui vont donc au-delà de relations interpersonnelles. Dans la perspective lesbienne féministe du corpus étudié, ces relations sont analysées en termes de rapports de domination, qui repose sur l'appropriation des femmes (appropriation du travail, appropriation par le mariage, etc.).

19. Pour Lorde cette dimension érotique ne doit surtout pas être évacuée puisqu'elle constitue un pouvoir nécessaire à l'affirmation de soi, que les femmes doivent se réapproprier. Voir Céline Perrin : «Sister Outsider d'Audre Lorde : la poésie et la colère » (2004).

20. "la dimension érotique du concept de lesbienne». Critique formulée notamment par Ann Ferguson dans «Patriarchy, Sexual Identity and the Sexual Revolution» (1981). Le mot « lesbian » est en italique dans le texte d'origine.

21. "Ces identités peuvent parfois être entrelacées, mais ce sont des héritages séparés et distincts, et à d'autres moments, elles peuvent être en conflit. " [trad. de l'autrice]

22. Cette distinction dont parle Nestle est une séparation de l'histoire lesbienne vis-à-vis de « l'histoire des femmes », mais aussi de l'histoire plus générale de «l'homosexualité ». (Corbman $2014: 5)$

23. Se penser comme " un peuple » ou se rattacher à des lignées est une pratique antérieure aux années 1970. Mireille Havet (1898-1932) évoque par exemple le «clan divin des femmes amoureuses" dans ses écrits. Le militantisme des années 1970-80 produit en revanche un tournant collectif de productions communautaires de savoirs sur les passés.

24. " contes, chant, danse, fiction historique, mythologie, histoires de femmes ignorées, recherche sur les lesbiennes connues et les communautés lesbiennes du passé - tout ce qui recrée l'histoire des lesbiennes sous forme écrite, orale ou visuelle " [trad. de l'autrice]

25. "The will to remember: The Lesbian Herstory Archives of New-York", titre de l'article de Nestle (1990).

26. "Mais la raison essentielle pour créer les archives était le silence de l'histoire patriarcale à notre sujet - les femmes qui ont aimé les femmes. » [trad. de l'autrice]

27. Pour les analyses biographiques, Doughty privilégie de décrire et comprendre "womanloving in terms of the culture in which it occurs, as best we can within our own cultural limits » (1979 : 77) ("l'amour des femmes pour les femmes, selon les termes employés dans la culture où il est décrit, de la meilleure façon dont nous le pouvons selon nos propres limites culturelles. " [trad. de l'autrice]), afin de ne pas imposer une définition universelle et atemporelle d'un terme « lesbienne » de plus en plus mobilisateur à mesure qu'il se politise.

28. Nous employons le terme d'euphémisation car il est, au même titre que "distorsion", mobilisé en anglais de manière récurrente dans les textes militants, afin de dénoncer les effets stylistiques visant à minorer soit une lesbianité «évidente», soit des relations féminines pourtant très développées par les autrices.

29. On notera que ces productions écrites limitent le champ des représentations aux lesbiennes de classes sociales circonscrites, certaines étant par ailleurs des écrivaines reconnues en leur 
temps (mais parfois moins qu'à titre posthume), dans des milieux sociaux et spatiaux où la culture écrite était la norme.

30. Déceler n'est pas affirmer. Comme elle le précise dans son article, ces « indices » doivent être analysés, entrecroisés et confrontés comme dans n'importe quelle entreprise scientifique : «as lesbian-feminist biographers, we need to establish the limits of the validity of the above set of signs in terms of distinctions of class, race, age, date, and geographical location. " (Doughty 1979 : 77). (« en tant que biographes lesbiennes-féministes, nous devons établir les limites de la validité de l'ensemble des signes ci-dessus en termes de distinctions de classe, de race, d'âge, de date et de situation géographique. » [trad. de l'autrice])

31. Il s'agit d'articuler un certain nombre de situations agissant comme « indices » à mettre en relation : un environnement particulièrement féminin; une exaltation des valeurs de l'amitié féminine; un travail centré sur la vie de l'autrice (introspectif); une préoccupation « inhabituelle »; un intérêt particulier pour les groupes opprimés ou déviants ; voire des amitiés avec des hommes homosexuels (Doughty 1979 : 77).

32. « Nos critères, et non les leurs, devraient prévaloir » [trad. de l'autrice]

33. "Les productions des femmes dans tous les domaines tendent à disparaitre des archives historiques; leurs productions créées dans des sphères uniquement valorisées par des femmes sont invisibles presque à coup sûr.» [trad. de l'autrice]

34. Pour Chamberland, les recherches historiques de Faderman s'inscrivent dans la vision du continuum de Rich, ne fournissant pas «les outils pour démarquer diverses pratiques de résistance " $(1989$ : 138). Selon elle, Faderman propose une « relecture intéressante des amitiés sentimentales féminines, qu'elle[s] assimile[nt] au lesbianisme", créant en revanche de la confusion en considérant comme « lesbien » ou féministe » les «manifestations de support entre les femmes» (138). Pour Rich cependant, les résistances des femmes au patriarcat ne sont pas nécessairement «consciemment féministes» au sens contemporain, mais une sorte de « underground river », une rivière souterraine qui alimente les rébellions (Schwarz [Rich] 1979 : 6) La critique de Chamberland fait toutefois écho à deux enjeux fortement débattus: l'assimilation des lesbiennes à toutes les femmes en lutte contre le patriarcat (ignorant ainsi leurs oppressions spécifiques) et la possibilité ou non d'apposer des termes et des intentions politiques contemporaines à des relations ayant eu lieu à des époques antérieures (à ce sujet, voir les réponses de différentes autrices à la Question 4 du questionnaire de Schwarz 1979).

35. Faderman analyse les biographies de Seward (1742-1809) et Dickinson (1830-1886) réalisées dans les années 1930 au spectre d'une relecture pathologique de l'homosexualité qui n'existait pas comme telle à la période d'écriture des écrivaines (les terminologies homosexuelle/ hétérosexuel-le n'étant d'ailleurs pas usitées). Les autrices pouvaient cependant être confrontées à des normes hétérocentriques sociales, temporelles et situées 監 notamment celle du mariage bien qu'aucune des deux ne se soit justement mariée.

36. " aux endroits où les biographes ont été trop scrupuleux pour expurger, ils ont néanmoins réussi à déformer l'histoire lesbienne en éludant l'évidence. " [trad. de l'autrice]

37. En italique et en français dans le texte original rédigé en anglais. Technique qui consiste, comme son nom l'indique, à « chercher l'homme » sans lequel les femmes «n'auraient pas pu » créer ou produire ces textes.

38. Ce qui est en revanche loin d'être évident selon les corpus : les matériaux n'ont pas toujours été rendus accessibles aux biographes ouvertement lesbiennes, surtout en l'absence d'affiliation à une «"respectable" institution such as a university » (Doughty1979 : 79).

39. "Position historique particulière d'avoir à prouver l'existence même de son objet. » [trad. de l'autrice] 40. « La charge de la preuve devrait incomber à l'autre interprétation - qu'elles ne soient pas lesbiennes.» [trad. de l'autrice]

41. Judith Schwarz était alors une chercheuse indépendante, qui a écrit aux Archives pour la première fois en 1977 et a rejoint le collectif deux années plus tard. Dans la référence citée, elle 
pose douze questions à différentes intervenantes sur leurs rapports aux archives et histoires lesbiennes, dont Nestle, Rich et Doughty. Ici, une des réponses de Nestle.

42. "faire confiance aux "savoirs historiques" ou aux "institutions universitaires/académiques" " [trad. de l'autrice]

43. La " herstorical vision » est une perspective analytique féministe de l'histoire. Le «His » (pronom dit masculin en anglais) de « histoire » est remplacée par «Her» (pronom dit féminin en anglais). Cela remet en question "l'his-toire» (comme histoire d'hommes), en mettant l'accent sur les rôles des femmes et se réappropriant cette histoire d'un point de vue féministe. Dans la Newsletter $n^{\circ} 5$, Sturgis écrit : « herstory is : not the wars and diplomatic dealings of a handful men, but ourselves, our lives, our interpretations of our experiences » (1979: 4) (« La "herstory" est: non pas les guerres et les relations diplomatiques d'une poignée d'hommes, mais nousmêmes, nos vies, nos interprétations de nos expériences. " [trad. de l'autrice])

44. "La "Lesbian Herstory Archives" doit rester dans sa communauté, non par chauvinisme mais par vision féministe-historique. Nous n'existons pas dans les savoirs historiques ou les institutions académiques, bien que nous y voyagions incognito. Nous vivons dans nos maisons, dans les rues, dans les bars, à nos bureaux, à notre travail, avec nos enfants, dans nos groupes, et nous créons notre histoire chaque jour." [trad. de l'autrice]

45. Les échanges et débats entre savoirs académiques et «contre-savoirs » militants demanderaient une étude à part entière. Pour approfondir, voir Weeks (2019) «Écrire l'histoire des sexualités ", notamment la sous-partie " Savoirs non officiels et contre-histoire ».

46. "Les archives devraient être hébergées au sein de la communauté, et non sur un campus qui est par définition fermé à de nombreuses femmes [...] Si nécessaire, les archives resteront dans la clandestinité avec leurs peuples pour être chéries dans des endroits secrets jusqu'à ce que la communauté soit en sécurité.» [trad. de l'autrice]

47. "Étudiantes, nous ne voulons pas que votre travail soit perdu. Nous voulons conserver dans nos dossiers tous les documents relatifs à tous les aspects de l'expérience lesbienne. Ne vous inquiétez pas du style ou de la note. Envoyez-nous une copie de votre travail afin que toutes les lesbiennes puissent partager votre découverte. » [trad. de l'autrice]

48. On trouve d'ailleurs une référence à ce propos dans la Newsletter $n^{\circ} 5$, dans la rubrique « From other women » qui regroupe des annonces de personnes ou groupes à propos de leurs recherches ou pratiques d'archivages. Le groupe "Circle of Lesbian Indexers» y présente son projet d'indexation de la «Lesbian culture » et appelle la "Lesbian community » à y participer, proposer ajouts ou corrections (1979: 5).

49. Pour notre analyse des pratiques archivistiques comprises entre 1975 et 1990, les termes "présent» ou «contemporaine » renverront, sauf indication contraire, au présent des militantes, à leurs productions « en direct ».

50. "Nous collecterons et conserverons TOUS les documents pertinents sur la vie et les expériences des lesbiennes : livres, magazines, revues, coupures de journaux (des médias institués, féministes et lesbiens), photos, bibliographies, informations historiques, cassettes audio, films, journaux intimes, histoires, poésie et prose, biographies, autobiographies, annonces d'événements, affiches, graphiques et autres souvenirs et obscures références à nos vies. " [trad. de l'autrice]

51. Weeks (2019), qui réalise dans ce chapitre une synthèse de plusieurs ouvrages, fait ici référence à la réflexion de Cvetkovich $(2003: 368)$.

52. «Les racines des Archives se trouvent dans les voix réduites au silence, les lettres d'amour détruites, les pronoms changés, les photos jamais prises, les distorsions euphémisées que le patriarcat aurait laissé passer. Pour mettre fin à l'héritage des visages perdus, nous demandons aux lesbiennes de tout le pays d'envoyer une photo d'elles-mêmes, d'amis, d'enfants, de maisons, d'animaux domestiques, d'activités, aux Archives. Aidez-nous à faire en sorte que nos futures sœurs puissent nous voir. » [trad. de l'autrice]

53. "Les livres, newsletters, pamphlets sont faciles à trouver, mais ce dont nous avons maintenant un besoin urgent, ce sont des histoires orales sur cassettes, des photographies de visages, de maisons, d'œuvres 
d'art, d'amis, des autobiographies, des journaux, des lettres, des journaux intimes » [Pour la transcription anglophone du corps de texte, nous avons conservé l'écriture originale du tapuscrit. En revanche, pour cette traduction, nous avons modifié la ponctuation afin de fluidifier la lecture.]

54. "On nous a toujours demandé: mais tu ne veux pas dire [qu'il faudrait archiver] mon travail, mes poèmes, mes lettres, ma photo ? Il y avait toujours de l'incrédulité devant notre affirmation que leur vie était ce qu'il y avait de plus important. Mais j'avais connu cette privation si cruellement dans ma propre vie que cette question a fait ressortir tout mon feu et mon amour - Oui, oui, vous êtes la lesbienne pour laquelle les archives existent, pour raconter et partager votre histoire. » [trad. de l'autrice]

55. Voir notamment les encarts de la Newsletter $n^{\circ} 4$ (1978:4)

56. Dans son ouvrage Klein (2019) précise que la pratique des archives orales s'est développée dès les années 1960 chez les historien'ne·s (bien qu'existant très antérieurement), lorsque leur intérêt s'est porté « sur des groupes sociaux jusqu'alors inexistants dans le discours historiques » (2019: 71). Dans cette perspective «l'histoire se fait alors productrice de ses sources [...] faisant appel à la mémoire des groupes étudiés" (Klein 2019: 71). L'archive orale n'est donc pas "en soi radicale " (Weeks 2019 : 194), mais correspond selon Weeks « à l'esprit du nouvel activisme et à l'histoire militante ", étant "implicitement collaborative et coopérative » et "créant des liens entre les générations et les différents individus » $(2019: 195)$.

57. Audre Lorde est une essayiste et poétesse américaine et militante des luttes contre les ségrégations raciales. Par ses analyses, elle apporte les bases d'une analyse intersectionnelle. Elle s'est particulièrement intéressée aux effets performatifs du langage et la nécessité de le maitriser pour exister et mettre fin à la «tyrannie du silence » $(1978: 13)$. C'est lors de cette intervention qu'elle formule "Your silence will not save you », reprise par les rédactrices de la revue pour illustrer le début des échanges.

58. Julia Stanley (ou Julia Penelope), est une linguiste, auteure et philosophe états-unienne, lesbienne séparatiste qui a particulièrement travaillé sur ces questions et produit en 1988 la première anthologie sur le séparatisme lesbien (après avoir été exclue d'université au début de sa carrière puis n'accédant pas aux promotions sous prétexte de recherches jugées trop étroites). Elle a notamment participé aux réunions de planification de la LHA.

59. Journal littéraire, théorique et artistique féministe lesbien, créé en 1976. Les numéros mettent en lien des sujets quotidiens lesbiens et féministes avec des contributions littéraires et artistiques. La thématique du numéro analysé est «The Transformation of Silence into Language and Action ».

60. "Je me suis retrouvée à raconter à une autre femme [terme au plus proche, mais "womon" est une manière féministe d'écrire "woman" sans le "man" qui signifie "homme"] l'histoire de mon coming out, l'histoire de comment et quand je suis devenue lesbienne, dans tous les sens où j'utilise maintenant ce mot [...] Je voulais qu'elle me comprenne comme je me comprends, et j'ai découvert dans ce récit que ma vie, mon coming out, était un récit de silences, le silence du déni, de la haine de soi, de la douleur. " [trad. de l'autrice]

61. Hampton a notamment légué en 1976 aux LHA des fonds issus de ses propres collections, ainsi que les échanges épistolaires conservés durant sa vie (Nestle 1993 : 2).

62. Ces enregistrements sont désormais disponibles sur leur site internet, mais le moyen de diffusion le plus large vers les lesbiennes ne pouvant venir les écouter était auparavant la transcription dans les newsletters. Celui de Hampton est retranscrit dans la Newsletter $n^{\circ} 7$ (1981).

63. "lire et d'entendre comment l'expérience [du coming out] a affecté d'autres lesbiennes " [trad. de l'autrice]

64. Cela dépendant fortement des rapports aux notions et idéaux de communautés, aux pratiques politiques, aux engagements différenciés dans les luttes intersectionnelles, et bien sûr de l'intérêt porté aux archives. Dans sa thèse Orr (2017) explore ces problématiques (https://surface.syr.edu/ etd/803/) 
65. "un passé simplifié et homogénéisé ne sera pas assez riche en idées, inspirations, actions ou images pour nourrir une communauté lesbienne future, diversifiée et fortifiée. " [trad. de l'autrice]

66. " la transformation du silence en langage et en action " [trad. de l'autrice]

67. " En tant que lesbienne, je comprends l'importance qu'occupe le langage dans ma vie. Avec le langage, je peux revendiquer des facettes de moi-même que j'ai nié, exprimer des idées qui ont longtemps été réprimées et taboues. Avec le langage, je peux définir ma vie comme réelle, et je peux agir pour changer ma vie.» [trad. de l'autrice]

68. «si les Archives sont ici pour mettre fin à notre perte de mémoire générationnelle, chaque femme Lesbienne doit y ajouter sa voix.» [trad. de l'autrice]

69. "Nous essayons d'éviter l'hypocrisie que serait de commémorer les lesbiennes du passé tout en exilant leurs représentantes vivantes au nom d'une histoire [herstory] sélective. " [trad. de l'autrice]

70. Cet encart apparait pour la première fois dans la Newsletter $n^{\circ} 4$ (1978), avant de devenir régulier dans les autres numéros.

71. "Nous avons besoin que les femmes nous disent si elles devraient faire partie des Archives. Aidez-nous à mettre fin aux silences, si ce n'est pas pour maintenant, du moins pour l'avenir. » [trad. de l'autrice]

72. "Il est de notre responsabilité de valider l'expérience lesbienne les unes pour les autres, car c'est à travers notre réjouissance collective, notre reconquête et notre renouveau que notre survie en tant que communauté lesbienne sera déterminée. " [trad. de l'autrice]

73. Selon Klein, une perspective critique de l'archivistique contemporaine, reposant notamment sur les perspectives de l'exploitation des archives, devrait d'ailleurs «fournir une base pour mettre en lumière la fonction politique des archives - et donc des archivistes » (2020).

74. « Notre conception des archives doit être différente ; nous sommes différentes. » [trad. de l'autrice]

75. "Et déjà les archives portent la trace de la manière dont nous changeons l'histoire.» [trad. de l'autrice]

76. Ces surgissements du passé dans le présent via l'exploitation des archives portent également à réflexion dans certains courants archivistiques et philosophiques, notamment inspirés des pensées de Walter Benjamin sur le «concept d'histoire » et la critique du temps linéaire. Les archives portent en elles des informations du passé, qui se réinterprètent au présent via une nécessaire anachronie («condition sous laquelle le passé est saisissable » (Klein 2019: 117. Elle effectue la synthèse de la réflexion de Didi-Huberman 2000 : 15-16)). L'exploitation des archives offre la possibilité d'une archivistique critique en replaçant le matériau dans l'espace social, révélant « la manière dont la société les comprend et se les approprie » (Klein 2020). Des allersretours nécessaires au cours desquels les gestes archivistiques "posés dans un Maintenant [un présent réminiscent] entrent en résonnance avec ceux de l'Autrefois [un passé en latence], laissant une trace pour l'avenir. » (Klein : 2020). L'archive pourrait être ici « une modalité d'inscription de soi dans le temps » (Klein : 2020). Nous préciserons que les termes avec majuscule dans la citation sont ceux empruntés à la pensée benjaminienne et sont porteurs de significations précises, analysées dans le chapitre «La pensée benjaminienne » (voir Klein 2019 : 115-152).

77. "Durant son voyage de plus de quarante ans, la Lesbian Herstory Archives a établi une pratique au potentiel queer [...] où des pratiques d'archivages radicales rencontrent une politique centrée sur les lesbiennes dans le but de réaliser quelque chose de plus. » [trad. de l'autrice]

78. «Une fois que les lesbiennes possèderont des générations d'histoire [herstory] vécues, elles changeront l'histoire par la force de leurs présences." [trad. de l'autrice; avec l'emploi de "herstory » et "history», on peut comprendre qu'une fois l'histoire lesbienne sera établie, les lesbiennes pourront changer l'histoire dans son ensemble.]

79. Notamment les poèmes de Hart Crane et les romans de Willa Cather.

80. Les Amazones sont, dans la mythologie grecque, issues des rives de la Mer Noire. Cette mythologie a tant imprégné les références culturelles occidentales que le terme a été élargi à tout groupe de femmes guerrières, dont les « Amazones d'Amazonie » nommées ainsi par les colons au XVIe siècle. 
81. Le collectif et sa revue sont canadiens (et francophones, ce qui leur donne une place particulière dans les échanges transnationaux sur les pratiques militantes), nous devons donc préciser que le contexte historique et national du lesbianisme radical diffère de celui des ÉtatsUnis, son émergence et développement étant soumis aux possibilités de luttes, aux contextes locaux et nationaux, aux échanges avec d'autres mouvements militants, etc. Nous nous baserons donc sur les éléments discursifs qui prédominent dans la définition que les militantes ont de leur lesbianisme radical via leur revue, à savoir notamment le désir que le «lesbianisme devienne quelque chose de politique " (Turcotte 2020) et la nécessité de "contrer l'absence d'espace de réflexion théorique et historique sur le lesbianisme » (Coulombe 2020). Dans cette perspective, le lesbianisme radical est aussi un « point de vue pour analyser le monde » (Coulombe 2020).

82. Première parution en 1982.

83. À écouter dans l'émission radiophonique «Toutes les lesbiennes en parlent » le 27/10/2020, produite par le Réseau des lesbiennes du Québec (RLQ) qui, d'après le descriptif « deviendra un contenu archivistique important pour les générations à venir, afin d'engendrer le dialogue entre les femmes LGBTQ+ de tous âges. »

84. " intéressée par le fait d'explorer les possibilités de créer un mode de vie Amazone intellectuel et agraire à la périphérie du "système" avec d'autres lesbiennes. » [trad. de l'autrice]

85. Expérience qu'elle a d'ailleurs concrétisée avec Stanley en 1975, en emportant les archives de l'appartement de Nestle de New York en Californie (avant que ces dernières ne réintègrent finalement New York). (Corbman $2014: 8$ )

86. Figure mythologique de Cassandre, « celle qui écarte les hommes ».

87. Bilitis est une lesbienne fictive contemporaine de Sappho, issue du recueil de poèmes de Louÿs « Les chansons de Bilitis » (1894)

88. L'occultation comme « constance la plus forte de l'existence lesbienne » (Chamberland 1989: 142) via la figure de Sappho se trouve aussi dans la littérature francophone chez Bonnet « [...] l'invisible Gomorrhe n'accède même pas à l'existence et se voit occulter jusqu'en Sappho ses rares références culturelles » (1981).

89. «depuis la destruction de la poésie de Sappho, notre histoire nous a prévenues que nous faisons partie des déshéritées. " [trad. de l'autrice]

90. Ce qui n'est bien entendu pas nécessairement le cas. Les références, si elles doivent s'établir, peuvent être faites autour d'autres figures, d'autres perspectives ou revendications politiques. Par ailleurs, les références, notamment aux Amazones, peuvent être interprétées de manières très différentes pour un même terme, en fonction des contextes géographiques, historiques et culturels.

91. Voir « LHA Survival literature collection » (Newsletter $n^{\circ} 7$ 1981)

92. "alors que nous revendiquons notre littérature, la poésie a été la voix principale des femmes pauvres, de la classe ouvrière et de femmes de Couleur [traduction la plus littérale, bien que "femme de Couleur" puisse être connoté en français.] "

93. L'œuvre de Dickinson est présente car l'ouvrage disponible est une anthologie de 1960.

94. Par ailleurs, il ne s'agit pas uniquement de poèmes écrits, les militantes encourageant également l'envoi de poèmes lus sur des cassettes. Elles précisent enfin que les poèmes peuvent être dans toutes les langues, y compris l'espagnol et le français (Nlt4 $1978: 5$ ).

95. "nous demandons aux autrices lesbiennes de nous envoyer des copies de leur travail. Nos fonds sont limités mais notre objectif est de préserver les voix des Lesbiennes et de mettre fin au silence générationnel. » [trad. de l'autrice]

96. Toutes deux collaborant d'après Alfonsi dès 1924 dans Inversion - une des premières revues militantes homosexuelles traitant notamment d'art, de littérature et d'histoire. Si elles ne sont pas nécessairement définies comme militantes lesbiennes, l'enjeu réside plutôt dans la portée que cette réappropriation historique et artistique peut avoir pour les militantes et/ou lesbiennes actuelles. 
97. "Les Archives sont à la fois une bibliothèque et un "album de famille": une tentative de préserver nos expériences vécues au-delà de notre génération et de renouer avec notre famille du passé.» [trad. de l'autrice]

98. "préserver les vies et les voix des femmes plus âgées tant qu'elles sont encore présentes parmi nous." [trad. de l'autrice]

99. "Souvent dans les journaux, un euphémisme pour parler de la mort d'une lesbienne est "il n'y a pas de survivante connue". Ce n'est pas vrai. Nous sommes les survivantes les unes des autres.» [trad. de l'autrice]

100. « les Archives dans leurs sens le plus profond sont un acte politique » [trad. de l'autrice]

101. "apporter leur propre expérience à la catégorie, "restructurant le sens de cette expérience individuelle" (Dubriwny 398) d'une solitaire ignorance à une indignation partagée. » [trad. de l'autrice]

102. "Les vies des femmes qui ont vécu à différentes époques, villes ou nations peuvent chacune se toucher. Les lesbiennes individuelles et les communautés lesbiennes ne doivent plus jamais être fragmentées ou isolées. » [trad. de l'autrice]

103. "nos archives, notre album de famille, notre bibliothèque, n'étaient pas principalement destinés aux universitaires mais pour toute femme lesbienne qui avait besoin d'une image ou d'un mot pour survivre au jour.» [trad. de l'autrice]

104. «que notre communauté ne puisse être ciblée par la répression sociétale. » [trad. de l'autrice]

105. "Ce diaporama est devenu notre principal outil d'organisation, notre moyen le plus puissant de lutter contre les sentiments de privation culturelle et d'isolement personnel. Cela nous a également permis de clarifier notre vision - qu'était une archive lesbienne, en quoi était-elle différente des archives traditionnelles, comment s'insérait-elle dans les luttes politiques de notre peuple. » [trad. de l'autrice]

106. Reine de Suède (dont le titre est «Roi de Suède ») de 1632 à 1654, qui fait par ailleurs l'objet d'un article dans la revue The Furies (1970: 10-11)

107. "Notre newsletter et nos affichages dans les librairies, les bibliothèques et les centres communautaires gai·es, notre participation dans les manifestations et les marches, tout cela montre clairement qu'une archive lesbienne participe à la création de la culture et du changement social aussi bien qu'elle agit comme protectrice de l'histoire de notre peuple. » [trad. de l'autrice]

108. Weeks (2019), qui réalise dans ce chapitre une synthèse de plusieurs ouvrages, fait ici référence à la réflexion de Doan (2013).

109. " une forme de respect et d'affection, démontrant la place importante de l'archive dans l'imaginaire populaire lesbien. » [trad. de l'autrice]

\section{RÉSUMÉS}

La question des archives occupe une place centrale dans les militantismes lesbiens des années 1970 et 1980 aux États-Unis, soulignant l'intérêt - pour des communautés en train de se construire - d'avoir accès à leurs passés pour s'auto-définir et affirmer leurs possibilités d'existences. Cet article aborde les pratiques archivistiques des militantes lesbiennes sous l'angle du désir de transmission qui les anime, à travers l'étude de textes militants produits entre 1975 et 1990. À travers ces discours, la nécessité d'une analyse lesbienne-féministe se dessine, afin d'œuvrer à la réappropriation des matériaux d'archives passés, formant des corpus vecteurs de nouvelles significations. Les productions d'archives en direct (écrites, orales, photographiques), encouragées par les militantes, sont parties prenantes d'une multiplication des traces 
d'existences lesbiennes, palliant les manques passés, investissant les espaces contemporains et créant des matériaux à transmettre pour les générations futures. Enfin, les connexions qui s'opèrent entre ces expériences lesbiennes trans-temporelles viennent consolider les processus de transmissions de passés, faisant dialoguer généalogies recréées et générations à venir.

The issue of archives occupies a central place in the Lesbian activists of the 1970s and 1980s in the United States, highlighting interest - in communities under construction - to have access to their past to self-define and affirm their existence possibilities. This article addresses the archival practices of lesbian activists from the angle of the desire for transmission that animates them through the studies of activist texts produced between 1975 and 1990. Through these militant speeches, the need for a lesbian-feminist analysis is emerging, to work for reappropriation of past archive materials, creating corpus vectors of new meanings. Live archive productions (written, oral, photographic) encouraged by activists are stakeholders of a multiplication of the traces of lesbian lives, which sweeps past shortfalls, investing contemporary spaces and creating materials to be transmitted for future generations. Finally, the connections that take place between these trans-temporal lesbian experiments consolidate past transmissions processes, makes genealogies recreated and generations dialogue.

\section{INDEX}

Thèmes : Recherches

Mots-clés : archives, connexions, communautés, lesbiennes, transmission

Keywords : archives, communities, connections, lesbians, transmissions

\section{AUTEUR}

\section{MATHILDE PETIT}

Mathilde Petit est doctorante en Sociologie en co-direction à l'Université Paris Nanterre et l'Université Paris Vincennes Saint-Denis. Ses recherches explorent la manière dont les pratiques collectives d'archives et de mémoires lesbiennes participent à la production de savoirs militants et s'inscrivent dans les luttes politiques contemporaines. 